\title{
Broad Scope Aminocyclization of Enynes with Cationic JohnPhos- Gold(I) Complex as the Catalyst
}

\author{
Ricarda Miller, ${ }^{\dagger}$ Javier Carreras, $^{\dagger}$ Michael E. Muratore, ${ }^{\dagger}$ Morgane Gaydou, $^{\dagger}$ Francesco Camponovo, ${ }^{\dagger}$ \\ and Antonio M. Echavarren*, ${ }^{\dagger}$,
${ }^{\dagger}$ Institute of Chemical Research of Catalonia (ICIQ), Barcelona Institute of Science and Technology, Av. Països Catalans 16, 43007 Tarragona, Spain
${ }^{\ddagger}$ Departament de Química Analítica i Química Orgànica, Universitat Rovira i Virgili, C/Marcel-lí Domingo s/n, 43007 Tarragona, \\ Spain
}

Supporting Information

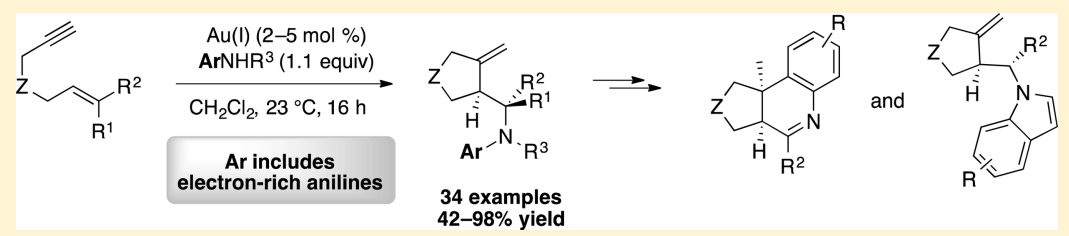

ABSTRACT: A practical aminocyclization of 1,6-enynes with a wide variety of substituted anilines, including $N$-alkyl anilines, has been achived by using cationic [JohnPhosAu(MeCN)] $\mathrm{SbF}_{6}$ as a general purpose catalyst. The resulting adducts can be easily converted into polycyclic compounds by palladium- and gold-catalyzed reactions.

\section{INTRODUCTION}

Gold(I) complexes are very active and selective catalysts for the addition of nucleophiles on 1,n-enynes. ${ }^{1,2}$ The most emblematic transformation in this area is the addition of alcohols and water on 1,6-enynes 1 (alkoxy- or hydroxycyclization), which occurs stereospecifically to form adducts 3 in the presence of gold(I) catalysts ${ }^{3,4}$ under much milder conditions than with other electrophilic metal catalysts (Scheme 1). ${ }^{5,6}$ According to

Scheme 1. Gold(I)-Catalyzed Alkoxy- or Hydroxycyclization of 1,6-Enynes

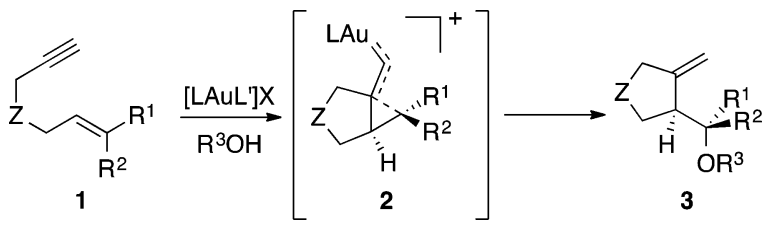

DFT calculations, this reaction proceeds through intermediates 2, whose structures are intermediate between cyclopropyl gold(I) carbenes and gold(I)-stabilized homoallyl carbocations. ${ }^{7}$ It is interesting to note that the hydroxycyclization is usually much faster than the direct nucleophilic addition of water on the terminal alkyne to form the corresponding methyl ketone. $^{8}$ Enantioselective hydroxy- and alkoxycyclizations of 1,6-enynes have been achieved with moderate to good enantioselectivities with a variety of chiral phosphines or NHC-gold catalysts. ${ }^{9}$ The hydroxy- and alkoxycyclizations of $1,5^{-4 b, 10,11}$ and 1,7-enynes take place similarly.

A few examples of intramolecular amination of 1,5-enynes have been described. ${ }^{10}$ The intermolecular reaction of 1,6- enynes with carbamates $\mathrm{ROCONH}_{2}(\mathrm{R}=\mathrm{Et}, \mathrm{Bn})$ and anilines also takes place using $\mathrm{Ph}_{3} \mathrm{PAuCl}$ and $\operatorname{AgSbF}_{6}(5 \mathrm{~mol} \%){ }^{13}$ However, in the latter case, only less basic anilines bearing strongly electron-withdrawing groups $\left(p-\mathrm{NO}_{2}, o-\mathrm{CN}, o-\mathrm{CF}_{3}, p-\right.$ $\mathrm{Cl}$ ) were used as the nucleophiles. We decided to study the scope and limitations of the intermolecular amination of 1,6enynes with a broader range of anilines using gold(I) complexes with JohnPhos and related bulky biphenyl-based phosphines (Buchwald ligands), which are often the catalysts of choice in gold(I)-catalyzed reactions. ${ }^{14}$

\section{RESULTS AND DISCUSSION}

The aminocyclization of enynes was initially studied with dimethyl 2-trans-cinnamyl-2-(prop-2-yn-1-yl)malonate (1a) as the substrate and a slight excess of the aniline using 2-5 mol \% of the gold catalyst in $\mathrm{CH}_{2} \mathrm{Cl}_{2}$ (Table 1). The best result for the addition of aniline was obtained using $2 \mathrm{~mol} \%$ of commercially available cationic JohnPhos-gold(I) catalyst A, leading to adduct $4 \mathbf{a}$ in excellent yield (Table 1 , entry 1 ). Increasing the catalyst loading to $5 \mathrm{~mol} \%$ was required for the addition of $p$ anisidine to form $4 \mathrm{~b}$ in $58 \%$ yield after a $19 \mathrm{~h}$ reaction time (Table 1 , entry 2 ). This result with an electron-rich aniline is remarkable since this reaction had only been reported with deactivated amines. ${ }^{13}$ Lower yields were obtained with gold(I) complexes with other phosphine, NHC, or phosphite ligands (Table 1, entries 3-7). Tetrahydrofuran was also evaluated as solvent in the reaction with aniline; however, the yield was significantly reduced. ${ }^{15}$

Received: November 12, 2015

Published: February 3, 2016 
Table 1. Gold(I)-Catalyzed Aminocyclization of 1,6-Enyne 1a

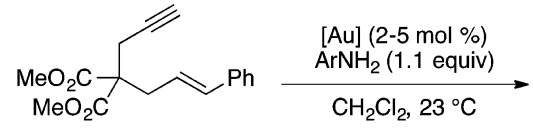

1a

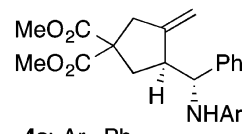

4a: $\mathrm{Ar}=\mathrm{Ph}$ 4b: $\mathrm{Ar}=p-\mathrm{MeOC}_{6} \mathrm{H}_{4}$

\begin{tabular}{cllcl} 
entry & \multicolumn{1}{c}{$\mathrm{Ar}$} & \multicolumn{1}{c}{$[\mathrm{Au}], \mathrm{mol} \%$} & $\begin{array}{c}\text { time } \\
(\mathrm{h})\end{array}$ & $\begin{array}{c}\text { product, yield } \\
(\%)^{a}\end{array}$ \\
1 & $\mathrm{Ph}$ & $\mathbf{A}(2)$ & 16 & $\mathbf{4 a}\left(95^{a}, 9^{b}\right)$ \\
2 & $p-\mathrm{MeOC}_{6} \mathrm{H}_{4}$ & $\mathbf{A}(5)$ & 19 & $\mathbf{4 b}(58)$ \\
3 & $p-\mathrm{MeOC}_{6} \mathrm{H}_{4}$ & $\mathbf{B}(5)$ & 19 & $\mathbf{4 b}(42)$ \\
4 & $p-\mathrm{MeOC}_{6} \mathrm{H}_{4}$ & $\mathrm{C}(5)$ & 19 & $\mathbf{4 b}(30)$ \\
5 & $p-\mathrm{MeOC}_{6} \mathrm{H}_{4}$ & $\mathrm{D}_{1}(5)$ & 19 & $\mathbf{4 b}(10)$ \\
6 & $p-\mathrm{MeOC}_{6} \mathrm{H}_{4}$ & $\mathbf{E}(5)$ & 19 & $\mathbf{4 b}(23)$ \\
7 & $p-\mathrm{MeOC}_{6} \mathrm{H}_{4}$ & $\mathrm{PPh}_{3} \mathrm{AuCl} / \mathrm{AgSbF}_{6}$ & 19 & $\mathbf{4 b}\left(7^{b}\right)$
\end{tabular}

${ }^{a}$ Yields determined by ${ }^{1} \mathrm{H}$ NMR. ${ }^{b}$ Isolated yield.
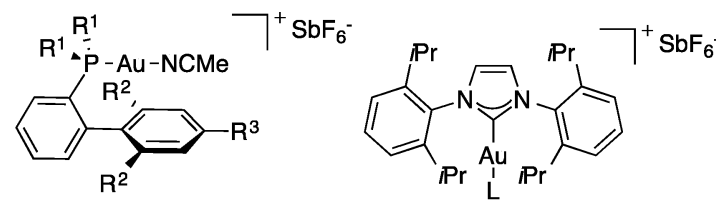

A: $R^{1}=t B u, R^{2}=R^{3}=H$

B: $R^{1}=C y, R^{2}=O M e, R^{3}=H$

$D_{1}: L=P h C N$

C: $R^{1}=t B u, R^{2}=R^{3}=\mathbb{P r}$

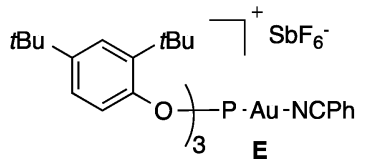

It is important to note that the reaction of anilines and aliphatic amines with complex $\mathbf{A}$ forms complexes [(JohnPhos) $\left.\mathrm{Au}\left(\mathrm{NH}_{2} \mathrm{R}\right)\right] \mathrm{SbF}_{6}$ by ligand substitution, which have been characterized by X-ray diffraction and show diminished catalytic activity in the reaction of cyclopropenes with $p$-anisidine. ${ }^{16}$ However, in our system, these less active amino complexes presumably formed in situ under the amination reaction still undergo ligand exchange with the enynes at a sufficient rate so as to generate the catalytically active $\left(\eta^{2}\right.$-alkyne) gold(I) species. ${ }^{15}$

The substrate generality under these reaction conditions was tested with different enynes and a wide range of anilines (Table 2). In general, moderate to excellent yields were obtained for the different combinations of 1,6-enynes and anilines examined (42-98\%). Concerning the anilines, electron-rich as well as electron-poor anilines participated efficiently in this reaction without significant differences in the isolated yields. Anilines substituted at the ortho-position also gave rise to the corresponding adducts $4 \mathrm{c}, 4 \mathrm{e}, 4 \mathrm{~h}, 4 \mathrm{o}, 4 \mathrm{w}$, and $4 \mathrm{y}$ in satisfactory yields. Secondary anilines, such as $\mathrm{N}$-methylaniline, $\mathrm{N}$-allylaniline, indoline, or tetrahydroquinoline also reacted to give the corresponding adducts in moderate to good yields (4l, 4t, 4adag). Different substitution patterns on the enyne were also used, and all led efficiently to the desired product. The presence of a dimethyl or phenyl substituent on the alkene did not give significantly different results in terms of reactivity. The relative configuration of the aminocyclization products was assigned by determining the structures of compounds $\mathbf{4 d}, \mathbf{4 i}$, and $\mathbf{4 x}$ by Xray crystallography analysis, ${ }^{17}$ confirming the anti-type addition of the nucleophile to the 1,6-enyne.
Table 2. Gold(I)-Catalyzed Aminocyclization of 1,6-Enynes la-f with Different Anilines
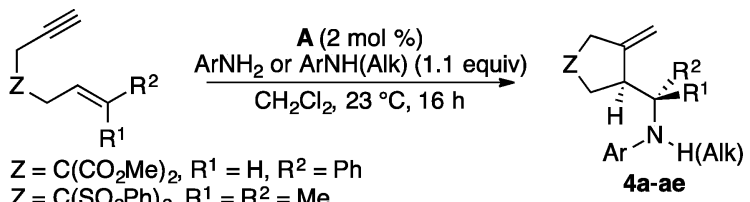

1a: $\mathrm{Z}=\mathrm{C}\left(\mathrm{CO}_{2} \mathrm{Me}\right)_{2}, \mathrm{R}^{1}=\mathrm{H}, \mathrm{R}^{2}=\mathrm{Ph}$

1b: $Z=C\left(S_{2} P h\right)_{2}, R^{1}=R^{2}=M e$

1c: $Z=C\left(\mathrm{CO}_{2} \mathrm{Me}\right)_{2}, \mathrm{R}^{1}=\mathrm{R}^{2}=\mathrm{Me}$

1d: $Z=2,2$-dimethyl-1,3-dioxane, $R^{1}=H, R^{2}=P h$

1e: $\mathrm{Z}=\mathrm{C}\left(\mathrm{CH}_{2} \mathrm{OPMB}\right)_{2}, \mathrm{R}^{1}=\mathrm{H}, \mathrm{R}^{2}=\mathrm{Ph}$

1f: $\mathrm{Z}=\mathrm{C}\left(\mathrm{CH}_{2} \mathrm{OMe}\right)_{2}, \mathrm{R}^{1}=\mathrm{H}, \mathrm{R}^{2}=\mathrm{Ph}$

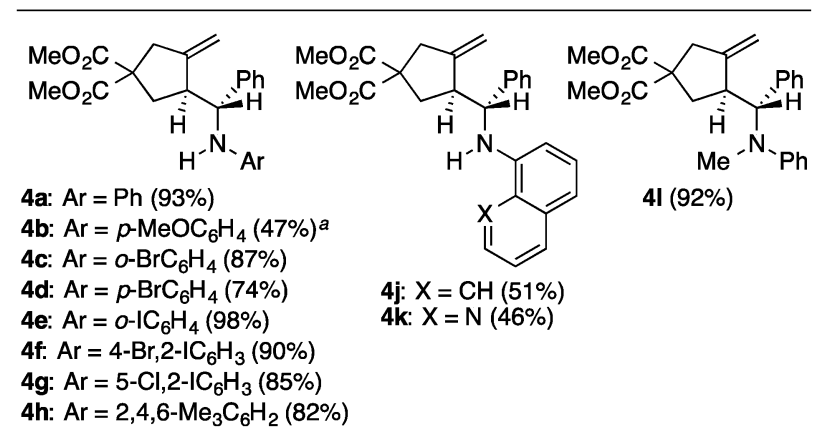

4i: $\mathrm{Ar}=3,5-\left(\mathrm{CF}_{3} \mathrm{C}_{6} \mathrm{H}_{3}(89 \%)\right.$
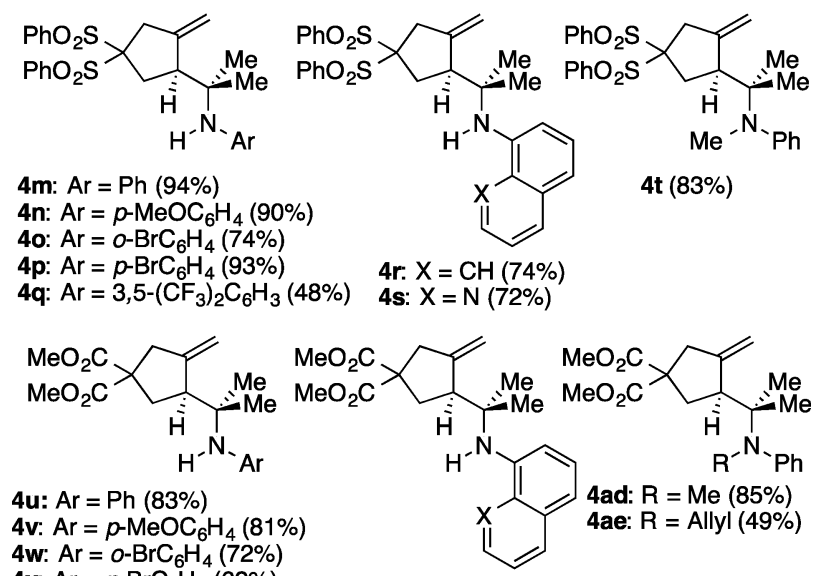

4w: $\mathrm{Ar}=0-\mathrm{BrC}_{6} \mathrm{H}_{4}(72 \%)$

4x: $\mathrm{Ar}=p-\mathrm{BrC}_{6} \mathrm{H}_{4}(62 \%)$

4y: $\mathrm{Ar}=0-\mathrm{IC}_{6} \mathrm{H}_{4}(58 \%)$

4z: $\mathrm{Ar}=p-\mathrm{MeCOC}_{6} \mathrm{H}_{4}(74 \%)$

4aa: $\mathrm{Ar}=3,5-\left(\mathrm{CF}_{3}\right)_{2} \mathrm{C}_{6} \mathrm{H}_{3}(42 \%)$

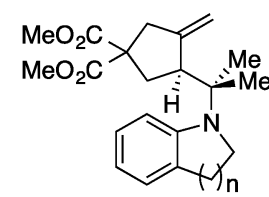

4af: $n=1(87 \%)$

4ag: $n=2(51 \%)$ 4ab: $\mathrm{X}=\mathrm{CH}(78 \%)$

4ac: $X=N(81 \%)$ $a_{5} \mathrm{~mol} \%$ catalyst.

To illustrate the synthetic potential of this gold(I) catalyzed reaction, additional transformations were carried out on several halogenated derivatives, in order to increase the molecular complexity. First, an intramolecular Heck reaction was successfully applied for compounds bearing iodine in the ortho position (Scheme 2, $\mathbf{4 e -}-\mathbf{g}$ ). Under standard conditions, good yields of the desired cis-fused tricyclic structures $5 \mathbf{e}-\mathbf{g}$ were obtained after selective 6-exo cyclization. This outcome represents an easy access to these tetrahydro- $1 \mathrm{H}$-cyclopenta$[c]$ quinoline derivatives. The use of $o-\mathrm{Br}$ derivative $4 \mathrm{c}$ or dimethyl substituted compound $4 \mathbf{y}$ only led to the recovery of the starting material. It is interesting to note that the initial 
Scheme 2. Transformation of Adducts $4 \mathrm{e}-\mathrm{g}$ into

Tetrahydro- $1 H$-cyclopenta $[c]$ quinoline and Indole Derivatives via $\mathrm{Pd}(0)$-Catalyzed Cross-Coupling and $\mathrm{Au}(\mathrm{I})$ Catalyzed Cyclization

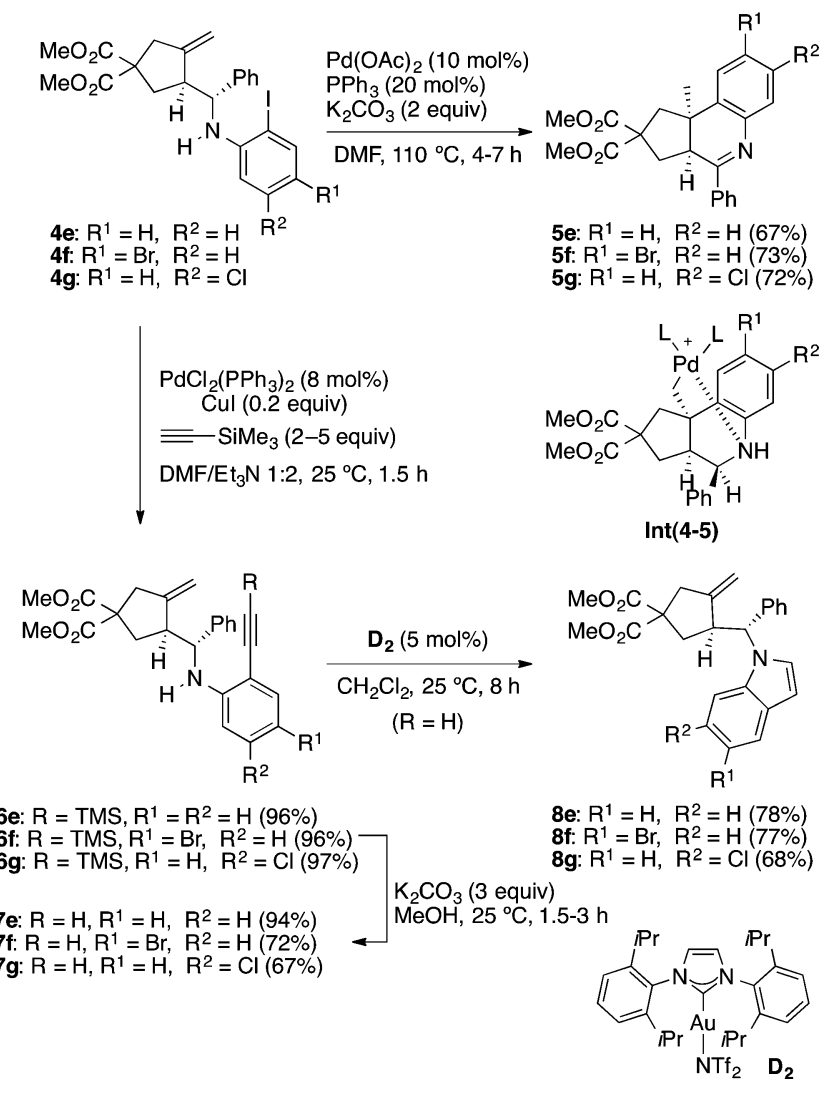

alkyl-palladium(II) species of the Heck reaction evolves by an intramolecular redox process presumably via $\beta$-hydride elimination from the benzylic position of cationic intermediate Int(4-5), followed by reductive elimination.

Then, we envisioned a second gold-catalyzed aminocyclization to form $\mathrm{N}$-substituted indoles (Scheme 2). The alkyne was introduced at the ortho position by Sonogashira cross-coupling of the iodoarenes with trimethylsilylacetylene to form $6 \mathrm{e}-\mathrm{g}$ in near-quantitative yields, followed by deprotection of the TMSalkynyl compounds by methanolysis to give $7 \mathrm{e}-\mathrm{g}$. The new gold(I)-catalyzed aminocyclization proceeded efficiently using catalyst $\mathbf{D}_{2}$ with IPr as the NHC ligand, leading to indole derivatives $\mathbf{8 e}-\mathbf{g}$ in good yields. The presence of chlorine or bromine atoms in $\mathbf{5} \mathbf{f}-\mathbf{g}$ and $\mathbf{8 f}-\mathbf{g}$ could allow their further functionalizations by cross-coupling reactions or other tranformations.

\section{CONCLUSIONS}

Although amines coordinate to Lewis acidic gold(I) complexes, thus reducing their availability to activate alkynes and other substrates, robust cationic gold(I) complex [JohnPhosAu$(\mathrm{MeCN})] \mathrm{SbF}_{6}$ is the catalyst of choice for the broad-scope aminocyclization of 1,6-enynes. The reaction proceeds under mild conditions in moderate to excellent yields with a wide variety of substituted anilines, including electron-rich as well as secondary $\mathrm{N}$-alkyl anilines to give the corresponding adducts, which can be further derivatized to form polycyclic compounds by palladium- and gold-catalyzed reactions.

\section{EXPERIMENTAL SECTION}

General Experimental Procedures. Solvents were dried by passing through an activated alumina column on a solvent purification system. Analytical thin layer chromatography was carried out using TLC-aluminum sheets with $0.2 \mathrm{~mm}$ of silica gel (Merck GF254) using UV light as the visualizing agent or an acidic solution of vanillin in ethanol as the developing agent. Purifications by chromatography were carried out using flash grade silica gel (SDS Chromatogel 60 ACC, $40-60 \mathrm{~mm})$. Preparative TLC was performed on $20 \mathrm{~cm} \times 20 \mathrm{~cm}$ silica gel plates $(2.0 \mathrm{~mm}$ thick, catalogue number 02015, Analtech). Organic solutions were concentrated under reduced pressure on a rotary evaporator. Unless otherwise stated, NMR spectra were recorded at $298 \mathrm{~K}$ on 300,400 , and $500 \mathrm{MHz}$ devices. ${ }^{1} \mathrm{H}$ and ${ }^{13} \mathrm{C}$ chemical shifts $(\delta)$ are given in ppm relative to TMS, and coupling constants $(J)$, in Hz. The solvent signals were used as references, and the chemical shifts were converted to the TMS scale. Mass spectra were recorded employing TOF mass analyzers (ESI, EI, CI). Melting points were determined by observation of the fusion of the solids placed in a capillary, through a magnifying glass. Crystal structure determinations were carried out using a diffractomer equipped with an APPEX $24 \mathrm{~K}$ CCD area detector, an FR591 rotating anode with Mo K $\alpha$ radiation, Montel mirrors as the monochromator, and a Kryoflex low temperature device $\left(T=-173{ }^{\circ} \mathrm{C}\right)$. Full-sphere data collection was used with $w$ and j scans. Programs used: Data collection APEX-2, data reduction Bruker Saint V/.60A and absorption correction SADABS. Structure Solution and Refinement: Crystal structure solution was achieved using direct methods as implemented in SHELXTL and visualized using the program XP. Missing atoms were subsequently located from difference Fourier synthesis and added to the atom list. Least-squares refinement on F2 using all measured intensities was carried out using the program SHELXTL. All non-hydrogen atoms were refined including anisotropic displacement parameters.

Compounds $\mathbf{1 a},{ }^{18} \mathbf{1 b},{ }^{19}$ and $\mathbf{1} \mathbf{c}^{19}$ were prepared according to reported methods. Gold complexes $\mathbf{A}, \mathbf{B}$, and $\mathbf{C}$ are commercialy available; $\mathbf{D}_{1}{ }^{2}{ }^{2} \mathbf{D}_{2}{ }^{4 \mathrm{C}}$ and $\mathrm{E}^{2}$ were prepared according to literature procedures.

2-Cinnamyl-2-(prop-2-yn-1-yl)propane-1,3-diol. $\mathrm{LiAlH}_{4}(461 \mathrm{mg}$, $12.1 \mathrm{mmol}$ ) was suspended in anhydrous THF $(30 \mathrm{~mL})$, and the slurry was cooled to $0{ }^{\circ} \mathrm{C}$. A solution of malonate $1 \mathrm{a}(1.58 \mathrm{~g}, 5.52 \mathrm{mmol})$ in anhydrous THF $(2 \mathrm{~mL})$ was added dropwise at $0{ }^{\circ} \mathrm{C}$. When effervescence had ceased, the mixture was allowed to warm to room temperature and was then heated at $45{ }^{\circ} \mathrm{C}$ and stirred vigorously for $3.5 \mathrm{~h}$. TLC (c-hexane/EtOAc 8:1 and 1:1) showed full consumption of the starting malonate and clean conversion. The mixture was diluted with wet diethyl ether $(30 \mathrm{~mL})$ and quenched by addition of sodium sulfate decahydrate (ca. $2 \mathrm{~g}$ ) slowly, to control the effervescence. After stirring for $1 \mathrm{~h}$ at $\mathrm{rt}$, a few drops of saturated aqueous $\mathrm{NH}_{4} \mathrm{Cl}$ were added, until the suspension became white. The solids were filtered off over Celite, and the cake was washed thoroughly with diethyl ether $(200 \mathrm{~mL})$. The filtrate was concentrated in vacuo, and the residue was purified by column chromatography on silica gel eluting with pentane/ diethyl ether 1:1 to straight diethyl ether to afford $1.15 \mathrm{~g}$ of a colorless solid (90\%). ${ }^{1} \mathbf{H}$ NMR $\left(500 \mathrm{MHz}, \mathrm{CDCl}_{3}\right) \delta 7.39-7.35(\mathrm{~m}, 2 \mathrm{H})$, $7.34-7.29(\mathrm{~m}, 2 \mathrm{H}), 7.25-7.21(\mathrm{~m}, 1 \mathrm{H}), 6.50(\mathrm{~d}, J=15.6 \mathrm{~Hz}, 1 \mathrm{H})$, $6.23(\mathrm{dt}, J=15.6,7.7 \mathrm{~Hz}, 1 \mathrm{H}), 3.76-3.67(\mathrm{~m}, 4 \mathrm{H}), 2.43-2.37(\mathrm{~m}$, $2 \mathrm{H}), 2.33(\mathrm{dd}, J=7.7,1.4 \mathrm{~Hz}, 2 \mathrm{H}), 2.32(\mathrm{~d}, J=2.8 \mathrm{~Hz}, 2 \mathrm{H}), 2.08(\mathrm{t}, J$ $=2.7 \mathrm{~Hz}, 1 \mathrm{H}) \mathrm{ppm} .{ }^{13} \mathrm{C}$ NMR $\left(126 \mathrm{MHz}, \mathrm{CDCl}_{3}\right) \delta 137.2,133.6$, $128.5,127.3,126.1,124.8,80.9,71.0,67.5,42.7,35.2,21.6 \mathrm{ppm}$. HRMS-APCI calcd for $\mathrm{C}_{15} \mathrm{H}_{19} \mathrm{O}_{2}^{+}[\mathrm{M}+\mathrm{H}]^{+}$: 231.1380; found: 231.1386. Mp 100-102 ${ }^{\circ} \mathrm{C}$.

5-Cinnamyl-2,2-dimethyl-5-(prop-2-yn-1-yl)-1,3-dioxane (1d). In a dry flask, 2-cinnamyl-2-(prop-2-yn-1-yl)propane-1,3-diol (600 mg, $2.61 \mathrm{mmol})$ was dissolved in anhydrous acetone $(2 \mathrm{~mL})$ and $p \mathrm{TsOH}$. $\mathrm{H}_{2} \mathrm{O}(25 \mathrm{mg}, 0.13 \mathrm{mmol})$ was added, followed by addition of $\mathrm{MgSO}_{4}$ $(300 \mathrm{mg}$ ). The suspension was stirred vigorously at rt for $24 \mathrm{~h}$. TLC showed clean conversion to a new product and only traces of starting material. Acetone was removed under a stream of nitrogen, and the mixture was loaded on silica gel and purified by column chromatography eluting with pentane/diethyl ether 95:5 to 9:1 to 
afford $650 \mathrm{mg}$ of a colorless oil (92\%). Remark: melting point close to room temperature. ${ }^{1} \mathbf{H}$ NMR $\left(500 \mathrm{MHz}, \mathrm{CDCl}_{3}\right) \delta 7.39-7.35(\mathrm{~m}$, $2 \mathrm{H}), 7.35-7.30(\mathrm{~m}, 2 \mathrm{H}), 7.26-7.21(\mathrm{~m}, 1 \mathrm{H}), 6.51(\mathrm{~d}, J=15.8 \mathrm{~Hz}$, $1 \mathrm{H}), 6.19(\mathrm{dt}, J=15.6,7.7 \mathrm{~Hz}, 1 \mathrm{H}), 3.73(\mathrm{~s}, 4 \mathrm{H}), 2.42(\mathrm{~d}, J=2.8 \mathrm{~Hz}$, $2 \mathrm{H}), 2.37(\mathrm{dd}, J=7.8,1.3 \mathrm{~Hz}, 2 \mathrm{H}), 2.08(\mathrm{t}, J=2.7 \mathrm{~Hz}, 1 \mathrm{H}), 1.46(\mathrm{~s}$, $3 \mathrm{H}), 1.44$ (s, 3H) ppm. ${ }^{13} \mathrm{C}$ NMR $\left(126 \mathrm{MHz}, \mathrm{CDCl}_{3}\right) \delta 137.2,133.9$, 128.5, 127.3, 126.1, 124.0, 98.2, 80.6, 71.1, 66.7, 36.1, 36.0, 25.3, 22.5, 22.2 ppm. HRMS-ESI calcd for $\mathrm{C}_{18} \mathrm{H}_{22} \mathrm{O}_{2} \mathrm{Na}^{+}[\mathrm{M}+\mathrm{Na}]^{+}: 293.1512$; found: 293.1512 .

(E)-(4,4-Bis(methoxymethyl)hept-1-en-6-yn-1-yl)benzene (1e). ${ }^{20}$ In a dry flask, 2-cinnamyl-2-(prop-2-yn-1-yl)propane-1,3-diol (200 $\mathrm{mg}, 0.87 \mathrm{mmol}$ ) was dissolved in anhydrous THF $(2 \mathrm{~mL})$, and $\mathrm{NaH}$ (80 mg, $2 \mathrm{mmol}), \mathrm{MeI}(135 \mathrm{~mL}, 2.17 \mathrm{mmol})$, and TBAI (48 mg, 0.13 $\mathrm{mmol}$ ) were added sequentially. The suspension was stirred vigorously at $\mathrm{rt}$ for $24 \mathrm{~h}$. TLC showed full consumption of starting material and conversion to diprotected alcohol with traces of monoprotection. The mixture was quenched by careful addition of saturated aqueous $\mathrm{NH}_{4} \mathrm{Cl}$ $(30 \mathrm{~mL})$ and extracted with diethyl ether $(3 \times 50 \mathrm{~mL})$. The combined organic layers were washed with brine $(2 \times 15 \mathrm{~mL})$, dried over $\mathrm{MgSO}_{4}$, and filtered, and the solvent was removed under vacuum. The resulting mixture was purified by column chromatography on silica gel eluting with c-hexane/EtOAc 10:1 to afford $210 \mathrm{mg}$ of a colorless oil (94\%). ${ }^{1} \mathrm{H}$ NMR $\left(500 \mathrm{MHz}, \mathrm{CDCl}_{3}\right) \delta 7.39-7.35(\mathrm{~m}, 2 \mathrm{H}), 7.34-7.29$ $(\mathrm{m}, 2 \mathrm{H}), 7.25-7.20(\mathrm{~m}, 1 \mathrm{H}), 6.47(\mathrm{~d}, J=15.8 \mathrm{~Hz}, 1 \mathrm{H}), 6.23(\mathrm{dt}, J=$ 15.6, $7.7 \mathrm{~Hz}, 1 \mathrm{H}), 3.36(\mathrm{~s}, 6 \mathrm{H}), 3.34-3.28(\mathrm{~m}, 4 \mathrm{H}), 2.34(\mathrm{dd}, J=7.7$, $1.3 \mathrm{~Hz}, 2 \mathrm{H}), 2.27(\mathrm{~d}, J=2.7 \mathrm{~Hz}, 2 \mathrm{H}), 2.02(\mathrm{t}, J=2.7 \mathrm{~Hz}, 1 \mathrm{H}) \mathrm{ppm}$. ${ }^{13} \mathrm{C}$ NMR $\left(126 \mathrm{MHz}, \mathrm{CDCl}_{3}\right) \delta 137.7,133.1,128.5,127.0,126.1$, $125.7,81.3,74.3,70.1,59.3,42.4,35.4,22.2$ ppm. HRMS-APCI calcd for $\mathrm{C}_{17} \mathrm{H}_{23} \mathrm{O}_{2}^{+}\left[\mathrm{M}+\mathrm{H}^{+}:\right.$: 259.1693; found: 259.1690 .

4,4'-(((2-Cinnamyl-2-(prop-2-yn-1-yl)propane-1,3diyl)bis(oxy))bis(methylene)) Bis(methoxybenzene) (1f). ${ }^{20}$ In a dry flask, 2cinnamyl-2-(prop-2-yn-1-yl)propane-1,3-diol $(200 \mathrm{mg}, 0.87 \mathrm{mmol})$ was dissolved in anhydrous THF $(2 \mathrm{~mL})$, and $\mathrm{NaH}(73 \mathrm{mg}, 1.82$ $\mathrm{mmol}$ ), PMB-Cl $(0.26 \mathrm{~mL}, 1.91 \mathrm{mmol})$, and TBAI $(48 \mathrm{mg}, 0.13$ $\mathrm{mmol}$ ) were added sequentially. The suspension was stirred vigorously at $\mathrm{rt}$ for $24 \mathrm{~h}$. TLC showed full consumption of starting material and conversion to mono- and diprotected alcohols (diprotection major). The mixture was quenched by careful addition of saturated aqueous $\mathrm{NH}_{4} \mathrm{Cl}(30 \mathrm{~mL})$ and extracted with diethyl ether $(3 \times 50 \mathrm{~mL})$. The combined organic layers were washed with brine $(2 \times 15 \mathrm{~mL})$, dried over $\mathrm{MgSO}_{4}$, and filtered, and the solvent was removed under vacuum. The resulting mixture was purified by column chromatography on silica gel eluting with c-hexane/EtOAc 10:1 to afford $230 \mathrm{mg}$ of a colorless oil (64\%). ${ }^{1} \mathbf{H}$ NMR $\left(500 \mathrm{MHz}, \mathrm{CDCl}_{3}\right) \delta 7.34-7.23(\mathrm{~m}$, $8 \mathrm{H}), 7.23-7.18(\mathrm{~m}, 1 \mathrm{H}), 6.89-6.84(\mathrm{~m}, 4 \mathrm{H}), 6.39(\mathrm{~d}, J=15.7 \mathrm{~Hz}$, $1 \mathrm{H}), 6.12(\mathrm{dt}, J=15.6,7.7 \mathrm{~Hz}, 1 \mathrm{H}), 4.45(\mathrm{~s}, 4 \mathrm{H}), 3.81(\mathrm{~s}, 6 \mathrm{H}), 3.42-$ $3.36(\mathrm{~m}, 4 \mathrm{H}), 2.35(\mathrm{dd}, J=7.7,1.3 \mathrm{~Hz}, 2 \mathrm{H}), 2.31(\mathrm{~d}, J=2.7 \mathrm{~Hz}, 2 \mathrm{H})$, $1.98(\mathrm{t}, J=2.7 \mathrm{~Hz}, 1 \mathrm{H}) \mathrm{ppm} .{ }^{13} \mathrm{C}$ NMR $\left(126 \mathrm{MHz}, \mathrm{CDCl}_{3}\right) \delta 159.0$, 137.7, 132.9, 130.8, 129.1, 128.4, 126.9, 126.1, 125.9, 113.7, 81.4, 72.9, 71.4, 70.2, 55.2, 42.6, 35.4, $22.4 \mathrm{ppm}$. HRMS-ESI calcd for $\mathrm{C}_{31} \mathrm{H}_{34} \mathrm{O}_{4} \mathrm{Na}^{+}[\mathrm{M}+\mathrm{Na}]^{+}$: 493.2349; found: 493.2362 .

General Procedure for the Aminocyclization of 1,6-Enynes (GP-I). Enyne ( 1 equiv) and aniline (1.1 equiv) were placed in a dry flask, and [JohnPhosAu(MeCN)] $\operatorname{SbF}_{6}(\mathrm{~A})(2 \mathrm{~mol} \%)$ was added as a solution in anhydrous $\mathrm{CH}_{2} \mathrm{Cl}_{2}$ (overall $0.15 \mathrm{M}$ ). The solution was stirred vigorously at $\mathrm{rt}$ for $16 \mathrm{~h}$.

Dimethyl 3-Methylene-4-(phenyl(phenylamino)methyl)cyclopentane-1,1-dicarboxylate (4a). Obtained using catalyst A $(2.2 \mathrm{mg}$, $2.8 \mu \mathrm{mol})$, enyne $1 \mathrm{a}\left(0.14 \mathrm{~mL}, 1.0 \mathrm{M}\right.$ in dry $\left.\mathrm{CH}_{2} \mathrm{Cl}_{2}, 0.14 \mathrm{mmol}\right)$, and aniline $\left(0.30 \mathrm{~mL}, 0.5 \mathrm{M}\right.$ in dry $\left.\mathrm{CH}_{2} \mathrm{Cl}_{2}, 0.154 \mathrm{mmol}\right)$ as the nucleophile in $\mathrm{CH}_{2} \mathrm{Cl}_{2}(0.6 \mathrm{~mL})$ according to General Procedure I. Preparative TLC (c-hexane/EtOAc 5:1) yielded $49 \mathrm{mg}$ (93\%) of the aminated product as a colorless oil. ${ }^{1} \mathbf{H}$ NMR $\left(400 \mathrm{MHz}, \mathrm{CDCl}_{3}\right) \delta$ $7.41-7.40(\mathrm{~m}, 2 \mathrm{H}), 7.39-7.36(\mathrm{~m}, 2 \mathrm{H}), 7.26(\mathrm{tt}, J=4.2,1.5 \mathrm{~Hz}, 1 \mathrm{H})$, $7.10(\mathrm{dd}, J=8.6,7.4 \mathrm{~Hz}, 2 \mathrm{H}), 6.67(\mathrm{tt}, J=7.4,1.2 \mathrm{~Hz}, 1 \mathrm{H}), 6.54(\mathrm{dd}, J$ $=8.6,1.2 \mathrm{~Hz}, 2 \mathrm{H}), 5.10-5.08(\mathrm{~m}, 1 \mathrm{H}), 4.80-4.78(\mathrm{~m}, 1 \mathrm{H}), 4.57(\mathrm{~d}, J$ $=5.0 \mathrm{~Hz}, 1 \mathrm{H}), 4.34(\mathrm{~s}, 1 \mathrm{H}), 3.78(\mathrm{~s}, 3 \mathrm{H}), 3.71(\mathrm{~s}, 3 \mathrm{H}), 3.16-3.07(\mathrm{~m}$, $2 \mathrm{H}), 3.01-2.96(\mathrm{~m}, 1 \mathrm{H}), 2.39-2.33(\mathrm{~m}, 1 \mathrm{H}), 2.28-2.24(\mathrm{~m}, 1 \mathrm{H})$ ppm. ${ }^{13} \mathrm{C}$ NMR $\left(101 \mathrm{MHz}, \mathrm{CDCl}_{3}\right) \delta 172.4,171.8,147.8,147.2$, $142.3,129.0,128.5,127.0,126.7,117.5,113.7,109.1,59.0,58.2$, 52.9,
52.8, 49.4, 42.0, 34.9 ppm. HRMS-ESI calcd for $\mathrm{C}_{23} \mathrm{H}_{26} \mathrm{NO}_{4}{ }^{+}[\mathrm{M}+$ $\mathrm{H}]^{+}$: 380.1856 ; found: 380.1865 .

Dimethyl 3-(((4-Methoxyphenyl)amino)(phenyl)methyl)-4methylenecyclopentane-1,1-dicarboxylate (4b). Obtained using catalyst A $(5.6 \mathrm{mg}, 7.4 \mu \mathrm{mol})$, enyne $1 \mathrm{a}(42 \mathrm{mg}, 0.15 \mathrm{mmol})$ and $p$ anisidine $(19 \mathrm{mg}, 0.16 \mathrm{~mol})$ as the nucleophile in $\mathrm{CH}_{2} \mathrm{Cl}_{2}(1.0 \mathrm{~mL})$ according to the General Procedure I. Purification by column chromatography ( $n$-hexane/EtOAc 9:1) yielded $28 \mathrm{mg}$ (47\%) of the aminated product as a yellow oil. ${ }^{1} \mathbf{H}$ NMR $\left(400 \mathrm{MHz}, \mathrm{CDCl}_{3}\right) \delta$ 7.38-7.18 (m, 5H), 6.70-6.65 (m, 2H), 6.47-6.42 (m, 2H), $5.06(\mathrm{br}$ s, $1 \mathrm{H}), 4.77($ br s, $1 \mathrm{H}), 4.44(\mathrm{~d}, J=4.6 \mathrm{~Hz}, 1 \mathrm{H}), 3.98(\mathrm{~s}, 1 \mathrm{H}), 3.73(\mathrm{~s}$, $3 \mathrm{H}), 3.67(\mathrm{~s}, 6 \mathrm{H}), 3.20-2.95(\mathrm{~m}, 3 \mathrm{H}), 2.32(\mathrm{dd}, J=13.6,9.8 \mathrm{~Hz}, 1 \mathrm{H})$, $2.23(\mathrm{dd}, J=13.6,8.7 \mathrm{~Hz}, 1 \mathrm{H}) \mathrm{ppm} .{ }^{13} \mathrm{C}$ NMR $\left(101 \mathrm{MHz}, \mathrm{CDCl}_{3}\right) \delta$ $172.4,172.0,152.2$, 147.6, 142.6, 142.2, 128.7, 127.1, 126.9, 115.1, $114.8,109.1,60.0,57.9,55.8,53.0,52.9,49.7,42.1,35.0$ ppm. HRMSESI calcd for $\mathrm{C}_{24} \mathrm{H}_{28} \mathrm{NO}_{5}{ }^{+}[\mathrm{M}+\mathrm{H}]^{+}: 410.1962$; found: 410.1984 .

Dimethyl 3-(((2-Bromophenyl)amino)(phenyl)methyl)-4methylenecyclopentane-1,1-dicarboxylate (4c). Obtained using catalyst A $(2.2 \mathrm{mg}, 2.8 \mu \mathrm{mol})$, enyne $1 \mathrm{a}(0.14 \mathrm{~mL}, 1.0 \mathrm{M}$ in dry $\left.\mathrm{CH}_{2} \mathrm{Cl}_{2}, 0.14 \mathrm{mmol}\right)$, and 2-bromoaniline $(26 \mathrm{mg}, 0.15 \mathrm{mmol})$ as the nucleophile in $\mathrm{CH}_{2} \mathrm{Cl}_{2}(0.85 \mathrm{~mL})$ according to General Procedure I. Preparative TLC (c-hexane/EtOAc 5:1) yielded $56 \mathrm{mg}$ (87\%) of the aminated product as a colorless oil. ${ }^{1} \mathbf{H}$ NMR $\left(400 \mathrm{MHz}, \mathrm{CDCl}_{3}\right) \delta$ $7.41(\mathrm{dd}, J=7.9,1.4 \mathrm{~Hz}, 1 \mathrm{H}), 7.35-7.31(\mathrm{~m}, 4 \mathrm{H}), 7.27-7.24(\mathrm{~m}$, $1 \mathrm{H}), 6.99(\mathrm{dt}, J=7.6,1.4 \mathrm{~Hz}, 1 \mathrm{H}), 6.53(\mathrm{dt}, J=7.6,1.4 \mathrm{~Hz}, 1 \mathrm{H}), 6.36$ (dd, $J=7.6,1.4 \mathrm{~Hz}, 1 \mathrm{H}), 5.09(\mathrm{~m}, 1 \mathrm{H}), 4.80(\mathrm{~d}, J=4.7 \mathrm{~Hz}, 1 \mathrm{H}), 4.72$ $(\mathrm{m}, 1 \mathrm{H}), 4.52(\mathrm{t}, J=5.3 \mathrm{~Hz}, 1 \mathrm{H}), 3.74(\mathrm{~s}, 3 \mathrm{H}), 3.71(\mathrm{~s}, 3 \mathrm{H}), 3.18-$ $3.16(\mathrm{~m}, 1 \mathrm{H}), 3.03(\mathrm{~s}, 2 \mathrm{H}), 2.45(\mathrm{dd}, J=13.7,8.2 \mathrm{~Hz}, 1 \mathrm{H}), 2.29(\mathrm{dd}, J$ $=13.7,9.9 \mathrm{~Hz}, 1 \mathrm{H}) \mathrm{ppm} .{ }^{13} \mathrm{C}$ NMR $\left(101 \mathrm{MHz}, \mathrm{CDCl}_{3}\right) \delta 171.8$, $171.7,146.9,144.3,141.2,132.2,128.5,128.2,127.3,126.8,118.1$, 112.9, 110.3, 109.9, 60.3, 58.1, 52.9, 52.8, 49.4, 42.1, 35.5 ppm. HRMS-ESI calcd for $\mathrm{C}_{23} \mathrm{H}_{25} \mathrm{BrNO}_{4}^{+}[\mathrm{M}+\mathrm{H}]^{+}:$458.0961; found: 458.0969 .

Dimethyl 3-(((4-Bromophenyl)amino)(phenyl)methyl)-4methylenecyclopentane-1,1-dicarboxylate (4d). Obtained using catalyst A (5.7 mg, $7.4 \mu \mathrm{mol})$, enyne $1 \mathrm{a}(42 \mathrm{mg}, 0.15 \mathrm{mmol})$, and 4-bromoaniline $(31 \mathrm{mg}, 0.18 \mathrm{~mol})$ as the nucleophile in $\mathrm{CH}_{2} \mathrm{Cl}_{2}(1.0$ $\mathrm{mL}$ ) according to General Procedure I. Purification by column chromatography (pentane/diethyl ether 9:1 to 4:1) yielded $50 \mathrm{mg}$ (74\%) of the aminated product as a white solid, which was further crystallized from $\mathrm{Et}_{2} \mathrm{O}$ /pentane. ${ }^{1} \mathrm{H}$ NMR $\left(400 \mathrm{MHz}, \mathrm{CDCl}_{3}\right) \delta 7.40-$ $7.22(\mathrm{~m}, 5 \mathrm{H}), 7.18-7.14(\mathrm{~m}, 2 \mathrm{H}), 6.43-6.36(\mathrm{~m}, 2 \mathrm{H}), 5.08(\mathrm{br} \mathrm{s}$, $1 \mathrm{H}), 4.78(\mathrm{br} \mathrm{s}, 1 \mathrm{H}), 4.55-4.50(\mathrm{~m}, 2 \mathrm{H}), 3.78(\mathrm{~s}, 3 \mathrm{H}), 3.71(\mathrm{~s}, 3 \mathrm{H})$, 3.14-3.05 (m, 2H), 2.99-2.92 (m, 1H), $2.35(\mathrm{dd}, J=14.0,9.3 \mathrm{~Hz}$, $1 \mathrm{H}), 2.21$ (dd, $J=14.0,9.3 \mathrm{~Hz}, 1 \mathrm{H}) \mathrm{ppm} .{ }^{13} \mathrm{C}$ NMR $(101 \mathrm{MHz}$, $\left.\mathrm{CDCl}_{3}\right) \delta 172.7,171.9,147.2,146.9,141.9,131.8,128.8,127.3,126.7$, $115.3,109.2$, 109.1, 58.9, 57.7, 53.1, 52.9, 49.3, 42.2, 34.7 ppm. HRMS-ESI calcd for $\mathrm{C}_{23} \mathrm{H}_{25} \mathrm{BrNO}_{4}{ }^{+}[\mathrm{M}+\mathrm{H}]^{+}$: 458.0961; found: 458.0971. Mp $124-126{ }^{\circ} \mathrm{C}$.

Dimethyl 3-(((2-lodophenyl)amino)(phenyl)methyl)-4methylenecyclopentane-1,1-dicarboxylate (4e). Obtained using catalyst A (15 mg, $20 \mu \mathrm{mol})$, enyne $1 \mathrm{la}(286 \mathrm{mg}, 1 \mathrm{mmol})$, and 2 iodoaniline $(234 \mathrm{mg}, 1.07 \mathrm{mmol})$ as the nucleophile in $\mathrm{CH}_{2} \mathrm{Cl}_{2}$ (4 $\mathrm{mL}$ ) according to General Procedure I. Purification by column chromatography (pentane/diethyl ether $9: 1$ to $4: 1$ ) yielded $489 \mathrm{mg}$ $(98 \%)$ of the aminated product as a colorless solid. Note: on 2.79 mmol scale, the reaction afforded the desired compound in $90 \%$ yield. ${ }^{1} \mathrm{H}$ NMR $\left(500 \mathrm{MHz}, \mathrm{CDCl}_{3}\right) \delta 7.64(\mathrm{dd}, J=7.8,1.5 \mathrm{~Hz}, 1 \mathrm{H}), 7.36-$ $7.30(\mathrm{~m}, 4 \mathrm{H}), 7.28-7.22(\mathrm{~m}, 1 \mathrm{H}), 7.00(\mathrm{ddd}, J=8.2,6.8,1.5 \mathrm{~Hz}, 1 \mathrm{H})$, 6.39 (ddd, $J=7.8,7.3,1.5 \mathrm{~Hz}, 1 \mathrm{H}), 6.27(\mathrm{dd}, J=8.2,1.5 \mathrm{~Hz}, 1 \mathrm{H}), 5.10$ $(\mathrm{q}, J=2.2 \mathrm{~Hz}, 1 \mathrm{H}), 4.76(\mathrm{q}, J=2.2 \mathrm{~Hz}, 1 \mathrm{H}), 4.61(\mathrm{~d}, J=4.6 \mathrm{~Hz}, 1 \mathrm{H})$, $4.52(\mathrm{t}, J=5.1 \mathrm{~Hz}, 1 \mathrm{H}), 3.73(\mathrm{~s}, 3 \mathrm{H}), 3.70(\mathrm{~s}, 3 \mathrm{H}), 3.16(\mathrm{dtt}, J=12.3$, $5.5,2.3 \mathrm{~Hz}, 1 \mathrm{H}), 3.11-2.99(\mathrm{~m}, 2 \mathrm{H}), 2.42(\mathrm{ddd}, J=13.7,8.1,1.4 \mathrm{~Hz}$, $1 \mathrm{H}), 2.28(\mathrm{dd}, J=13.7,10.1 \mathrm{~Hz}, 1 \mathrm{H}) \mathrm{ppm} .{ }^{13} \mathrm{C}$ NMR $(126 \mathrm{MHz}$, $\left.\mathrm{CDCl}_{3}\right) \delta 171.8,171.7,147.0,146.4,141.1,138.8,129.1,128.6,127.3$, $126.8,119.0,112.2,110.0,86.2,60.7,58.2,52.9,52.8,49.5,42.1,35.5$ ppm. HRMS-ESI calcd for $\mathrm{C}_{23} \mathrm{H}_{24} \mathrm{INO}_{4} \mathrm{Na}^{+}[\mathrm{M}+\mathrm{Na}]^{+}: 528.0642$, found 528.0637. Mp $129-131{ }^{\circ} \mathrm{C}$.

Dimethyl 3-(((4-Bromo-2-iodophenyl)amino)(phenyl)methyl)-4methylenecyclopentane-1,1-dicarboxylate (4f). Obtained using 
catalyst A (19 mg, $24 \mu \mathrm{mol})$, enyne $1 \mathrm{a}(350 \mathrm{mg}, 1.22 \mathrm{mmol})$, and 4 bromo-2-iodoaniline (401 $\mathrm{mg}, 1.35 \mathrm{mmol}$ ) as the nucleophile in $\mathrm{CH}_{2} \mathrm{Cl}_{2}(8 \mathrm{~mL})$ according to General Procedure I. Purification by column chromatography (pentane/ $\mathrm{Et}_{2} \mathrm{O}$ 9:1) yielded $644 \mathrm{mg}(90 \%)$ of the aminated product as a white solid. ${ }^{1} \mathrm{H}$ NMR $\left(500 \mathrm{MHz}, \mathrm{CDCl}_{3}\right)$ $\delta 7.72(\mathrm{~d}, J=2.3 \mathrm{~Hz}, 1 \mathrm{H}), 7.35-7.21(\mathrm{~m}, 5 \mathrm{H}), 7.07(\mathrm{dd}, J=8.7,2.3$ $\mathrm{Hz}, 1 \mathrm{H}), 6.11(\mathrm{~d}, J=8.7 \mathrm{~Hz}, 1 \mathrm{H}), 5.10(\mathrm{q}, J=2.2 \mathrm{~Hz}, 1 \mathrm{H}), 4.74(\mathrm{q}, J$ $=2.2 \mathrm{~Hz}, 1 \mathrm{H}), 4.61(\mathrm{~d}, J=4.5 \mathrm{~Hz}, 1 \mathrm{H}), 4.47(\mathrm{t}, J=5.0 \mathrm{~Hz}, 1 \mathrm{H}), 3.72$ $(\mathrm{s}, 3 \mathrm{H}), 3.69(\mathrm{~s}, 3 \mathrm{H}), 3.18-3.11(\mathrm{~m}, 1 \mathrm{H}), 3.03(\mathrm{bs}, 2 \mathrm{H}), 2.39$ (dd, $J=$ 13.7, 8.2 Hz, 1H), $2.24(\mathrm{dd}, J=13.7,10.2 \mathrm{~Hz}, 1 \mathrm{H}) \mathrm{ppm} .{ }^{13} \mathrm{C}$ NMR $\left(126 \mathrm{MHz}, \mathrm{CDCl}_{3}\right) \delta 171.9,171.8,147.1,145.8,140.7,140.3,132.0$, $128.8,127.7,126.9,113.3,110.3,109.4,86.3,60.9,58.3,53.1,53.0$, 49.5, 42.3, 35.6 ppm. HRMS-ESI calcd for $\mathrm{C}_{23} \mathrm{H}_{23} \mathrm{BrINO}_{4} \mathrm{Na}^{+}[\mathrm{M}+$ $\mathrm{Na}]^{+}$: 605.9747; found: 605.9747. Mp $123-125^{\circ} \mathrm{C}$.

Dimethyl 3-(((5-Chloro-2-iodophenyl)amino)(phenyl)methyl)-4methylenecyclopentane-1,1-dicarboxylate (4g). Obtained using catalyst A (19 mg, $24 \mu \mathrm{mol})$, enyne $1 \mathrm{a}(350 \mathrm{mg}, 1.22 \mathrm{mmol})$, and 5-chloro-2-iodoaniline $(341 \mathrm{mg}, 1.35 \mathrm{mmol}$ ) as the nucleophile in $\mathrm{CH}_{2} \mathrm{Cl}_{2}(8 \mathrm{~mL})$ according to General Procedure I. Purification by column chromatography (pentane $/ \mathrm{Et}_{2} \mathrm{O} 9: 1$ ) yielded $562 \mathrm{mg}(85 \%)$ of the aminated product as a white solid. ${ }^{1} \mathrm{H}$ NMR $\left(500 \mathrm{MHz}, \mathrm{CDCl}_{3}\right)$ $\delta 7.51(\mathrm{~d}, J=8.3 \mathrm{~Hz}, 1 \mathrm{H}), 7.37-7.29(\mathrm{~m}, 4 \mathrm{H}), 7.28-7.23(\mathrm{~m}, 1 \mathrm{H})$, $6.39(\mathrm{dd}, J=8.3,2.3 \mathrm{~Hz}, 1 \mathrm{H}), 6.24(\mathrm{~d}, J=2.3 \mathrm{~Hz}, 1 \mathrm{H}), 5.09(\mathrm{q}, J=2.1$ $\mathrm{Hz}, 1 \mathrm{H}), 4.73(\mathrm{q}, J=2.2 \mathrm{~Hz}, 1 \mathrm{H}), 4.68(\mathrm{~d}, J=5.0 \mathrm{~Hz}, 1 \mathrm{H}), 4.49(\mathrm{t}, J=$ $5.3 \mathrm{~Hz}, 1 \mathrm{H}), 3.72(\mathrm{~s}, 3 \mathrm{H}), 3.69(\mathrm{~s}, 3 \mathrm{H}), 3.20-3.12(\mathrm{~m}, 1 \mathrm{H}), 3.05-2.99$ $(\mathrm{m}, 2 \mathrm{H}), 2.42(\mathrm{dd}, J=13.7,8.1 \mathrm{~Hz}, 1 \mathrm{H}), 2.23(\mathrm{dd}, J=13.7,10.1 \mathrm{~Hz}$, 1H) ppm. ${ }^{13} \mathrm{C}$ NMR $\left(126 \mathrm{MHz}, \mathrm{CDCl}_{3}\right) \delta 171.9,171.8,147.5,147.0$, 140.5, 139.4, 135.4, 128.9, 127.7, 126.9, 119.1, 112.2, 110.3, 83.2, 60.7, 58.3, 53.1, 53.0, 49.4, 42.3, 35.6 ppm. HRMS-ESI calcd for $\mathrm{C}_{23} \mathrm{H}_{23} \mathrm{ClINO}_{4} \mathrm{Na}^{+}[\mathrm{M}+\mathrm{Na}]^{+}:$562.0253; found: 562.0262. Mp $143-145{ }^{\circ} \mathrm{C}$.

Dimethyl 3-((Mesitylamino)(phenyl)methyl)-4-methylenecyclopentane-1,1-dicarboxylate (4h). Obtained using catalyst A $(2.2 \mathrm{mg}$, $2.8 \mu \mathrm{mol})$, enyne $1 \mathrm{a}\left(0.14 \mathrm{~mL}, 1.0 \mathrm{M}\right.$ in dry $\left.\mathrm{CH}_{2} \mathrm{Cl}_{2}, 0.14 \mathrm{mmol}\right)$, and 2,4,6-trimethylaniline $\left(0.31 \mathrm{~mL}, 0.5 \mathrm{M}\right.$ in $\left.\mathrm{CH}_{2} \mathrm{Cl}_{2}, 0.15 \mathrm{mmol}\right)$ as the nucleophile in $\mathrm{CH}_{2} \mathrm{Cl}_{2}(0.6 \mathrm{~mL})$ according to General Procedure I. Preparative TLC (c-hexane/EtOAc 5:1) yielded $48 \mathrm{mg}$ (82\%) of the aminated product as a colorless oil. ${ }^{1} \mathbf{H}$ NMR $\left(400 \mathrm{MHz}, \mathrm{CDCl}_{3}\right) \delta$ $7.21-7.14(\mathrm{~m}, 5 \mathrm{H}), 6.68(\mathrm{~s}, 2 \mathrm{H}), 4.86(\mathrm{~d}, J=1.7 \mathrm{~Hz}, 1 \mathrm{H}), 4.36(\mathrm{~d}, J=$ $1.7 \mathrm{~Hz}, 1 \mathrm{H}), 4.22(\mathrm{~d}, J=8.4 \mathrm{~Hz}, 1 \mathrm{H}), 3.77(\mathrm{~s}, 3 \mathrm{H}), 3.75(\mathrm{~s}, 3 \mathrm{H}), 3.45$ $(\mathrm{s}, 1 \mathrm{H}), 3.29-3.25(\mathrm{~m}, 1 \mathrm{H}), 2.98-2.87(\mathrm{~m}, 3 \mathrm{H}), 2.42(\mathrm{dd}, J=13.8$, $9.5 \mathrm{~Hz}, 1 \mathrm{H}), 2.16(\mathrm{~s}, 3 \mathrm{H}), 2.10(\mathrm{~s}, 6 \mathrm{H}) \mathrm{ppm} .{ }^{13} \mathrm{C}$ NMR $(101 \mathrm{MHz}$, $\left.\mathrm{CDCl}_{3}\right) \delta 172.2,172.0,148.2,142.8,141.5,130.2,129.6,128.4,128.0$ 127.4, 127.0, 110.0, 64.0, 58.1, 52.9, 52.8, 47.9, 42.2, 37.8, 20.4, 19.3 ppm. HRMS-ESI calcd for $\mathrm{C}_{26} \mathrm{H}_{32} \mathrm{NO}_{4}{ }^{+}[\mathrm{M}+\mathrm{H}]^{+}$: 422.2326; found: 422.2331 .

Dimethyl 3-(((3,5-Bis(trifluoromethyl)phenyl)amino)(phenyl)methyl)-4-methylenecyclopentane-1,1-dicarboxylate (4i). Obtained using catalyst A (5.7 mg, $7.4 \mu \mathrm{mol})$, enyne $1 \mathrm{a}(41 \mathrm{mg}, 0.14 \mathrm{mmol})$ and 3,5-bistrifluoromethylaniline $(25 \mu \mathrm{L}, 0.16 \mathrm{~mol})$ as the nucleophile in $\mathrm{CH}_{2} \mathrm{Cl}_{2}(1.0 \mathrm{~mL})$ according to General Procedure I. Purification by column chromatography (c-hexane/EtOAc 12:1) yielded $67 \mathrm{mg}$ (89\%) of the aminated product as a white solid. ${ }^{1} \mathbf{H}$ NMR (400 $\left.\mathrm{MHz}, \mathrm{CDCl}_{3}\right) \delta 7.36-7.30(\mathrm{~m}, 4 \mathrm{H}), 7.30-7.23(\mathrm{~m}, 1 \mathrm{H}), 7.07$ (br s, $1 \mathrm{H}), 6.85-6.81(\mathrm{~m}, 2 \mathrm{H}), 5.27(\mathrm{~d}, J=4.6 \mathrm{~Hz}, 1 \mathrm{H}), 5.10-5.05(\mathrm{~m}$, $1 \mathrm{H}), 4.79-4.76(\mathrm{~m}, 1 \mathrm{H}), 4.62(\mathrm{dd}, J=4.6 \mathrm{~Hz}, 1 \mathrm{H}), 3.78(\mathrm{~s}, 3 \mathrm{H}), 3.69$ $(\mathrm{s}, 3 \mathrm{H}), 3.20-3.13(\mathrm{~m}, 1 \mathrm{H}), 3.13-3.08(\mathrm{~m}, 1 \mathrm{H}), 2.93-2.86(\mathrm{~m}, 1 \mathrm{H})$, $2.39(\mathrm{dd}, J=14.2,8.5 \mathrm{~Hz}, 1 \mathrm{H}), 2.18(\mathrm{dd}, J=14.6,10.4 \mathrm{~Hz}, 1 \mathrm{H}) \mathrm{ppm}$. ${ }^{13} \mathrm{C}$ NMR (101 MHz, $\left.\mathrm{CDCl}_{3}\right) \delta 173.2,171.7,148.6,147.0,140.7$, $132.2(\mathrm{q}, J=32.9 \mathrm{~Hz}), 129.1,127.8,126.6,123.6(\mathrm{q}, J=273.4 \mathrm{~Hz})$, $112.7,110.3$ (sept, $J=4.1 \mathrm{~Hz}$ ), 109.2, 58.4, 57.6, 53.4, 53.0, 48.8, 42.4, 34.4 ppm. ${ }^{19} \mathrm{~F}$ NMR $\left(376.49 \mathrm{MHz}, \mathrm{CDCl}_{3}\right) \delta-63.0 \mathrm{ppm}$. HRMS-ESI calcd for $\mathrm{C}_{25} \mathrm{H}_{23} \mathrm{~F}_{6} \mathrm{NO}_{4} \mathrm{Na}^{+}[\mathrm{M}+\mathrm{Na}]^{+}$: 538.1423; found: 538.1437. Mp $143-145{ }^{\circ} \mathrm{C}$.

Dimethyl 3-Methylene-4-((naphthalen-1-ylamino)(phenyl)methyl)cyclopentane-1,1-dicarboxylate (4j). Obtained using catalyst A $(2.2 \mathrm{mg}, 2.8 \mu \mathrm{mol})$, enyne $1 \mathrm{a}\left(0.14 \mathrm{~mL}, 1.0 \mathrm{M}\right.$ in dry $\mathrm{CH}_{2} \mathrm{Cl}_{2}, 0.14$ $\mathrm{mmol})$, and naphtalen-1-amine $(20 \mathrm{mg}, 0.14 \mathrm{mmol})$ as the nucleophile in $\mathrm{CH}_{2} \mathrm{Cl}_{2}(0.8 \mathrm{~mL})$ according to General Procedure I. Preparative TLC (c-hexane/EtOAc 5:1) yielded $31 \mathrm{mg}(51 \%)$ of the aminated product as a colorless oil. ${ }^{1} \mathrm{H}$ NMR $\left(400 \mathrm{MHz}, \mathrm{CDCl}_{3}\right) \delta 8.10(\mathrm{br} \mathrm{d}, J$ $=7.9 \mathrm{~Hz}, 1 \mathrm{H}), 7.80(\mathrm{dd}, J=7.6,1.8 \mathrm{~Hz}, 1 \mathrm{H}), 7.54-7.44(\mathrm{~m}, 4 \mathrm{H}), 7.34$ $(\mathrm{t}, J=7.2 \mathrm{~Hz}, 2 \mathrm{H}), 7.28-7.24(\mathrm{~m}, 1 \mathrm{H}), 7.21-7.14(\mathrm{~m}, 2 \mathrm{H}), 6.33(\mathrm{dd}$, $J=7.2,1.2 \mathrm{~Hz}, 1 \mathrm{H}), 5.09(\mathrm{~d}, J=2.2 \mathrm{~Hz}, 1 \mathrm{H}), 4.85(\mathrm{~d}, J=2.2 \mathrm{~Hz}, 1 \mathrm{H})$, $4.80(\mathrm{~d}, J=4.7 \mathrm{~Hz}, 1 \mathrm{H}), 3.82(\mathrm{~s}, 3 \mathrm{H}), 3.73(\mathrm{~s}, 3 \mathrm{H}), 3.32-3.28(\mathrm{~m}$, $1 \mathrm{H}), 3.15(\mathrm{~d}, J=16.2 \mathrm{~Hz}, 1 \mathrm{H}), 3.02(\mathrm{dd}, J=16.2,1.9 \mathrm{~Hz}, 1 \mathrm{H}), 2.58$ $(\mathrm{dd}, J=14.0,9.6 \mathrm{~Hz}, 1 \mathrm{H}), 2.30(\mathrm{dd}, J=14.0,9.6 \mathrm{~Hz}, 1 \mathrm{H}) \mathrm{ppm} .{ }^{13} \mathrm{C}$ NMR $\left(101 \mathrm{MHz}, \mathrm{CDCl}_{3}\right) \delta 172.5,171.8,147.2,142.6,141.9,134.2$, 128.6, 127.1, 126.6, 126.4, 125.6, 124.7, 123.9, 120.2, 117.5, 109.1, 106.4, 58.7, 57.6, 52.9, 52.7, 49.4, 42.3, $34.7 \mathrm{ppm}$. HRMS-ESI calcd for $\mathrm{C}_{27} \mathrm{H}_{28} \mathrm{NO}_{4}^{+}[\mathrm{M}+\mathrm{H}]^{+}: 430.2013$; found: 430.2020 .

Dimethyl 3-Methylene-4-(phenyl(quinolin-8-ylamino)methyl)cyclopentane-1,1-dicarboxylate (4k). Obtained using catalyst A $(2.2 \mathrm{mg}, 2.8 \mu \mathrm{mol})$, enyne $1 \mathrm{a}\left(0.14 \mathrm{~mL}, 1.0 \mathrm{M}\right.$ in dry $\mathrm{CH}_{2} \mathrm{Cl}_{2}, 0.14$ $\mathrm{mmol})$, and quinolin-8-amine $(20 \mathrm{mg}, 0.14 \mathrm{mmol})$ as the nucleophile in $\mathrm{CH}_{2} \mathrm{Cl}_{2}(0.8 \mathrm{~mL})$ according to General Procedure I. Preparative TLC (c-hexane/EtOAc 5:1) yielded $28 \mathrm{mg}$ (46\%) of the aminated product as a colorless oil. ${ }^{1} \mathbf{H}$ NMR $\left(400 \mathrm{MHz}, \mathrm{CDCl}_{3}\right) \delta 8.79(\mathrm{dd}, J=$ $4.0,1.6 \mathrm{~Hz}, 1 \mathrm{H}), 8.05(\mathrm{dd}, J=8.0,1.6 \mathrm{~Hz}, 1 \mathrm{H}), 7.43(\mathrm{~d}, J=8.4 \mathrm{~Hz}$, $2 \mathrm{H}), 7.38(\mathrm{dd}, J=8.0,4.0 \mathrm{~Hz}, 1 \mathrm{H}), 7.32(\mathrm{t}, J=7.2 \mathrm{~Hz}, 2 \mathrm{H}), 7.24-7.19$ $(\mathrm{m}, 2 \mathrm{H}), 7.01(\mathrm{dd}, J=8.4,0.8 \mathrm{~Hz}, 1 \mathrm{H}), 6.78(\mathrm{~d}, J=6.0 \mathrm{~Hz}, 1 \mathrm{H}), 6.45$ (dd, $J=7.6,0.8 \mathrm{~Hz}, 1 \mathrm{H}), 5.01(\mathrm{~d}, J=2.0 \mathrm{~Hz}, 1 \mathrm{H}), 4.62(\mathrm{t}, J=6.4 \mathrm{~Hz}$, $1 \mathrm{H}), 4.58(\mathrm{~d}, J=2.0 \mathrm{~Hz}, 1 \mathrm{H}), 3.74(\mathrm{~s}, 3 \mathrm{H}), 3.73(\mathrm{~s}, 3 \mathrm{H}), 3.29-3.25$ (m, $1 \mathrm{H}), 3.13(\mathrm{qd}, J=16.4,2.4 \mathrm{~Hz}, 1 \mathrm{H}), 3.01(\mathrm{qd}, J=16.4,2.4 \mathrm{~Hz}$, $1 \mathrm{H}), 2.63$ (ddd. $J=14.0,9.6,1.6, \mathrm{~Hz}, 1 \mathrm{H}), 2.39(\mathrm{dd}, J=14.0,9.6 \mathrm{~Hz}$, $1 \mathrm{H}) \mathrm{ppm} .{ }^{13} \mathrm{C}$ NMR $\left(101 \mathrm{MHz}, \mathrm{CDCl}_{3}\right) \delta 171.9,171.8,147.1,147.0$, 143.9, 142.0, 138.4, 135.9, 128.5, 128.4, 127.5, 127.2, 127.1, 121.3, $114.2,109.9,106.2,60.3,58.4,52.8,49.4,41.9,36.2$ ppm. HRMS-ESI calcd for $\mathrm{C}_{26} \mathrm{H}_{27} \mathrm{~N}_{2} \mathrm{O}_{4}^{+}[\mathrm{M}+\mathrm{H}]^{+}$: 431.1965; found: 431.1954.

Dimethyl 3-((Methyl(phenyl)amino)(phenyl)methyl)-4methylenecyclopentane-1,1-dicarboxylate (4I). Obtained using catalyst A $(2.2 \mathrm{mg}, 2.8 \mu \mathrm{mol})$, enyne $1 \mathrm{a}(0.14 \mathrm{~mL}, 1.0 \mathrm{M}$ in dry $\mathrm{CH}_{2} \mathrm{Cl}_{2}, 0.14 \mathrm{mmol}$ ), and $\mathrm{N}$-methylaniline $(0.3 \mathrm{~mL}, 0.5 \mathrm{M}$ in dry $\left.\mathrm{CH}_{2} \mathrm{Cl}_{2}, 0.15 \mathrm{mmol}\right)$ as the nucleophile in $\mathrm{CH}_{2} \mathrm{Cl}_{2}(0.6 \mathrm{~mL})$ according to General Procedure I. Preparative TLC (c-hexane/EtOAc 5:1) yielded $50 \mathrm{mg}(92 \%)$ of the aminated product as a colorless oil. ${ }^{1} \mathbf{H}$ NMR $\left(400 \mathrm{MHz}, \mathrm{CDCl}_{3}\right) \delta 7.27-7.23(\mathrm{~m}, 7 \mathrm{H}), 6.89-6.86(\mathrm{~m}, 2 \mathrm{H})$, $6.75(\mathrm{tt}, J=7.2,1.0 \mathrm{~Hz}, 1 \mathrm{H}), 4.86-4.81(\mathrm{~m}, 2 \mathrm{H}), 4.22(\mathrm{~d}, J=2.1 \mathrm{~Hz}$, $1 \mathrm{H}), 3.79(\mathrm{~s}, 3 \mathrm{H}), 3.70(\mathrm{~s}, 3 \mathrm{H}), 3.60-3.57(\mathrm{~m}, 1 \mathrm{H}), 3.24(\mathrm{qd}, J=16.5$, $2.1 \mathrm{~Hz}, 1 \mathrm{H}), 3.01(\mathrm{dd}, J=16.6,2.1 \mathrm{~Hz}, 1 \mathrm{H}), 2.74$ (ddd, $J=13.5,7.7$, $1.5 \mathrm{~Hz}, 1 \mathrm{H}), 2.67(\mathrm{~s}, 3 \mathrm{H}), 2.12(\mathrm{dd}, J=13.5,9.9 \mathrm{~Hz}, 1 \mathrm{H}) \mathrm{ppm}^{13} \mathrm{C}$ NMR $\left(101 \mathrm{MHz}, \mathrm{CDCl}_{3}\right) \delta 172.4,171.9,150.1,148.6,138.9,129.2$, 128.1, 128.0, 127.3, 117.3, 113.9, 109.8, 65.7, 57.6, 52.9, 52.8, 43.4, 41.8, 38.6, 31.8 ppm. HRMS-ESI calcd for $\mathrm{C}_{24} \mathrm{H}_{28} \mathrm{NO}_{4}{ }^{+}[\mathrm{M}+\mathrm{H}]^{+}$: 394.2013; found: 394.2026 .

N-(2-(2-Methylene-4,4-bis(phenylsulfonyl)cyclopentyl)propan-2yl)aniline (4m). Obtained using catalyst A $(1.9 \mathrm{mg}, 2.5 \mu \mathrm{mol})$, enyne $1 \mathbf{b}\left(0.12 \mathrm{~mL}, 1.0 \mathrm{M}\right.$ in dry $\left.\mathrm{CH}_{2} \mathrm{Cl}_{2}, 0.12 \mathrm{mmol}\right)$, and aniline $(0.25 \mathrm{~mL}$, $0.5 \mathrm{M}$ in $\left.\mathrm{CH}_{2} \mathrm{Cl}_{2}, 0.124 \mathrm{mmol}\right)$ as the nucleophile in $\mathrm{CH}_{2} \mathrm{Cl}_{2}(0.6 \mathrm{~mL})$ according to General Procedure I. Preparative TLC (c-hexane/EtOAc 5:1) yielded $58 \mathrm{mg}$ (94\%) of the aminated product as a colorless oil. ${ }^{1}$ H NMR (400 MHz, CDCl $) \delta 8.09(\mathrm{dd}, J=6.4,0.8 \mathrm{~Hz}, 2 \mathrm{H}), 7.97$ $(\mathrm{dd}, J=5.6,0.8 \mathrm{~Hz}, 2 \mathrm{H}), 7.75-7.69(\mathrm{~m}, 2 \mathrm{H}), 7.62(\mathrm{t}, J=6.0 \mathrm{~Hz}, 2 \mathrm{H})$, $7.54(\mathrm{t}, J=6.4 \mathrm{~Hz}, 2 \mathrm{H}), 7.19(\mathrm{dd}, J=6.8,6.0 \mathrm{~Hz}, 2 \mathrm{H}), 6.81(\mathrm{t}, J=6.0$ $\mathrm{Hz}, 2 \mathrm{H}), 6.68(\mathrm{dd}, J=7.2,6.8 \mathrm{~Hz}, 1 \mathrm{H}), 5.02(\mathrm{br} \mathrm{s}, 2 \mathrm{H}), 3.63(\mathrm{br} \mathrm{s}$, $1 \mathrm{H}), 3.55(\mathrm{qd}, J=14.0,2.0 \mathrm{~Hz}, 1 \mathrm{H}), 3.35(\mathrm{dt}, J=14.0,2.0 \mathrm{~Hz}, 1 \mathrm{H})$, $2.91-2.82(\mathrm{~m}, 2 \mathrm{H}), 2.67(\mathrm{ddd}, J=12.4,7.2,1.2, \mathrm{~Hz}, 1 \mathrm{H}), 1.39(\mathrm{~s}$, $3 \mathrm{H}), 1.30(\mathrm{~s}, 3 \mathrm{H}) \mathrm{ppm} .{ }^{13} \mathrm{C}$ NMR $\left(101 \mathrm{MHz}, \mathrm{CDCl}_{3}\right) \delta 146.6,145.8$, 137.2, 135.8, 134.7, 134.5, 131.0, 130.9, 129.2, 128.7, 128.6, 118.5, $116.8,111.3,91.7,56.4,50.2,41.0,33.6,26.2,25.4$ ppm. HRMS-ESI calcd for $\mathrm{C}_{27} \mathrm{H}_{30} \mathrm{NO}_{4} \mathrm{~S}_{2}{ }^{+}[\mathrm{M}+\mathrm{H}]^{+}$: 496.1611; found: 496.1638 .

4-Methoxy-N-(2-(2-methylene-4,4-bis(phenylsulfonyl)cyclopentyl)propan-2-yl)aniline (4n). Obtained using catalyst A (1.9 $\mathrm{mg}, 2.5 \mu \mathrm{mol})$, enyne $1 \mathbf{b}\left(0.12 \mathrm{~mL}, 1.0 \mathrm{M}\right.$ in dry $\left.\mathrm{CH}_{2} \mathrm{Cl}_{2}, 0.12 \mathrm{mmol}\right)$, and 4-methoxyaniline $(15 \mathrm{mg}, 0.12 \mathrm{mmol})$ as the nucleophile in $\mathrm{CH}_{2} \mathrm{Cl}_{2}(0.8 \mathrm{~mL})$ according to General Procedure I. Preparative TLC (c-hexane/EtOAc 5:1) yielded $59 \mathrm{mg}(90 \%)$ of the aminated product as a colorless oil. ${ }^{1} \mathbf{H}$ NMR $\left(400 \mathrm{MHz}, \mathrm{CDCl}_{3}\right) \delta 8.10(\mathrm{dd}, J=8.0,0.8$ $\mathrm{Hz}, 2 \mathrm{H}), 7.99(\mathrm{dd}, J=8.4,1.2 \mathrm{~Hz}, 2 \mathrm{H}), 7.75-7.71(\mathrm{~m}, 2 \mathrm{H}), 7.62(\mathrm{t}, J$ $=8.0 \mathrm{~Hz}, 2 \mathrm{H}), 7.55(\mathrm{t}, J=8.0 \mathrm{~Hz}, 2 \mathrm{H}), 6.78(\mathrm{~d}, J=9.2 \mathrm{~Hz}, 2 \mathrm{H}), 6.72$ 
$(\mathrm{d}, J=9.2 \mathrm{~Hz}, 2 \mathrm{H}), 5.08(\mathrm{~s}, 1 \mathrm{H}), 5.04(\mathrm{~s}, 1 \mathrm{H}), 3.80(\mathrm{~s}, 3 \mathrm{H}), 3.56(\mathrm{qd}, J$ $=17.2,2.4 \mathrm{~Hz}, 1 \mathrm{H}), 3.16(\mathrm{dt}, J=8.4,2.0 \mathrm{~Hz}, 1 \mathrm{H}), 2.92-2.83(\mathrm{~m}, 2 \mathrm{H})$, 2.69 (ddd, $J=16.8,8.8,1.2, \mathrm{~Hz}, 1 \mathrm{H}), 1.27(\mathrm{~s}, 3 \mathrm{H}), 1.20(\mathrm{~s}, 3 \mathrm{H}) \mathrm{ppm}$. ${ }^{13}$ C NMR (101 MHz, $\left.\mathrm{CDCl}_{3}\right) \delta 153.9,146.8,139.1,137.2,135.9$, 134.6, 134.5, 131.0, 131.0, 128.7, 128.6, 121.4, 114.4, 111.4, 91.8, 57.0, $55.6,51.3,40.9,33.8,26.2,25.3 \mathrm{ppm}$. HRMS-ESI calcd for $\mathrm{C}_{28} \mathrm{H}_{32} \mathrm{NO}_{5} \mathrm{~S}_{2}^{+}[\mathrm{M}+\mathrm{H}]^{+}$: 526.1716; found: 526.1726 .

2-Bromo-N-(2-(2-methylene-4,4-bis(phenylsulfonyl)cyclopentyl)propan-2-yl)aniline (4o). Obtained using catalyst A (1.9 mg, 2.5 $\mu \mathrm{mol})$, enyne $1 \mathbf{b}\left(0.12 \mathrm{~mL}, 1.0 \mathrm{M}\right.$ in dry $\left.\mathrm{CH}_{2} \mathrm{Cl}_{2}, 0.12 \mathrm{mmol}\right)$, and 2bromoaniline $(21 \mathrm{mg}, 0.124 \mathrm{mmol})$ as the nucleophile in $\mathrm{CH}_{2} \mathrm{Cl}_{2}(0.8$ $\mathrm{mL}$ ) according to General Procedure I. Preparative TLC (c-hexane/ EtOAc 5:1) yielded $53 \mathrm{mg}(74 \%)$ of the aminated product as a colorless solid. ${ }^{1} \mathrm{H}$ NMR $\left(400 \mathrm{MHz}, \mathrm{CDCl}_{3}\right) \delta 8.11$ (dd, $J=8.8,1.2$ $\mathrm{Hz}, 2 \mathrm{H}), 8.01(\mathrm{dd}, J=8.8,1.2 \mathrm{~Hz}, 2 \mathrm{H}), 7.74-7.70(\mathrm{~m}, 2 \mathrm{H}), 7.63-$ $7.54(\mathrm{~m}, 4 \mathrm{H}), 7.46(\mathrm{dd}, J=7.6,1.2 \mathrm{~Hz}, 1 \mathrm{H}), 7.15(\mathrm{dt}, J=8.4,2.0 \mathrm{~Hz}$, $1 \mathrm{H}), 6.90(\mathrm{dd}, J=8.4,2.4 \mathrm{~Hz}, 1 \mathrm{H}), 6.61(\mathrm{dt}, J=8.0,1.2 \mathrm{~Hz}, 1 \mathrm{H}), 5.06$ $(\mathrm{s}, 1 \mathrm{H}), 5.00(\mathrm{~s}, 1 \mathrm{H}), 4.43(\mathrm{br} \mathrm{s}, 1 \mathrm{H}), 3.61(\mathrm{qd}, J=17.2,2.4 \mathrm{~Hz}, 1 \mathrm{H})$, $3.47(\mathrm{t}, J=7.6 \mathrm{~Hz}, 1 \mathrm{H}), 2.84(\mathrm{~d}, J=17.6 \mathrm{~Hz}, 1 \mathrm{H}), 2.73(\mathrm{dd}, J=15.6$, $8.4 \mathrm{~Hz}, 1 \mathrm{H}), 2.58(\mathrm{ddd}, J=15.6,9.2,1.6 \mathrm{~Hz}, 1 \mathrm{H}), 1.49(\mathrm{~s}, 3 \mathrm{H}), 1.34$ $(\mathrm{s}, 3 \mathrm{H})$ ppm. ${ }^{13} \mathrm{C}$ NMR $\left(101 \mathrm{MHz}, \mathrm{CDCl}_{3}\right) \delta 146.4,142.7,137.0$, $135.7,134.7,134.6,132.9,131.1,131.0,128.7,128.7,128.2,118.5$, 114.5 , 111.9, 111.5, 91.7, 56.7, 49.4, 40.7, 33.7, 26.2, 25.2 ppm. HRMS-ESI calcd for $\mathrm{C}_{27} \mathrm{H}_{28} \mathrm{BrNO}_{4} \mathrm{~S}_{2} \mathrm{Na}^{+}[\mathrm{M}+\mathrm{Na}]^{+}$: 596.0535; found: 596.0566. $\mathrm{Mp} 191-192{ }^{\circ} \mathrm{C}$.

4-Bromo-N-(2-(2-methylene-4,4-bis(phenylsulfonyl)cyclopentyl)propan-2-yl)aniline (4p). Obtained using catalyst A (1.9 mg, 2.5 $\mu \mathrm{mol})$, enyne $1 \mathbf{b}\left(0.12 \mathrm{~mL}, 1.0 \mathrm{M}\right.$ in dry $\left.\mathrm{CH}_{2} \mathrm{Cl}_{2}, 0.124 \mathrm{mmol}\right)$, and 4bromoaniline ( $21.4 \mathrm{mg}, 0.12 \mathrm{mmol})$ as the nucleophile in $\mathrm{CH}_{2} \mathrm{Cl}_{2}(0.8$ $\mathrm{mL}$ ) according to General Procedure I. Preparative TLC (c-hexane/ EtOAc 5:1) yielded $67 \mathrm{mg}$ (93\%) of the aminated product as a colorless foam. ${ }^{1} \mathbf{H}$ NMR $\left(400 \mathrm{MHz}, \mathrm{CDCl}_{3}\right) \delta 8.07$ (dd, $J=8.4,1.2$ $\mathrm{Hz}, 2 \mathrm{H}), 7.05(\mathrm{dd}, J=8.4,1.2 \mathrm{~Hz}, 2 \mathrm{H}), 7.76-7.70(\mathrm{~m}, 2 \mathrm{H}), 7.64-$ $7.53(\mathrm{~m}, 4 \mathrm{H}), 7.27-7.25(\mathrm{~m}, 2 \mathrm{H}), 6.58-6.55(\mathrm{~m}, 2 \mathrm{H}), 5.03(\mathrm{~s}, 1 \mathrm{H})$, $5.00(\mathrm{~s}, 1 \mathrm{H}), 3.70(\mathrm{br} \mathrm{s}, 1 \mathrm{H}), 3.53(\mathrm{qd}, J=17.2,2.4 \mathrm{~Hz}, 1 \mathrm{H}), 3.32(\mathrm{dt}$, $J=8.4,2.4 \mathrm{~Hz}, 1 \mathrm{H}), 2.87(\mathrm{~d}, J=17.2 \mathrm{~Hz}, 1 \mathrm{H}), 2.79$ (dd, $J=15.6,8.8$, $\mathrm{Hz} 1 \mathrm{H}), 2.62$ (ddd, $J=15.6,8.8,1.6 \mathrm{~Hz}, 1 \mathrm{H}), 1.48(\mathrm{~s}, 3 \mathrm{H}), 1.33(\mathrm{~s}$, $3 \mathrm{H}) \mathrm{ppm} .{ }^{13} \mathrm{C}$ NMR $\left(101 \mathrm{MHz}, \mathrm{CDCl}_{3}\right) \delta 146.5,144.9,137.1,135.7$, $134.7,134.6,132.0,131.9,131.0,128.7,128.6,118.1,116.7,111.3$, 91.6, 56.5, 49.9, 41.0, 33.5, 26.1, 25.3 ppm. HRMS-ESI calcd for $\mathrm{C}_{27} \mathrm{H}_{27} \mathrm{BrNO}_{4} \mathrm{~S}_{2}^{-}[\mathrm{M}-\mathrm{H}]^{-}:$572.0570; found: 572.0593. Mp 116$118{ }^{\circ} \mathrm{C}$.

$\mathrm{N}$-(2-(2-Methylene-4,4-bis(phenylsulfonyl)cyclopentyl)propan-2yl)-3,5-bis(trifluoromethyl)aniline (4q). Obtained using catalyst A (1.9 mg, $2.5 \mu \mathrm{mol})$, enyne $1 \mathbf{b}\left(0.12 \mathrm{~mL}, 1.0 \mathrm{M}\right.$ in dry $\mathrm{CH}_{2} \mathrm{Cl}_{2}, 0.12$

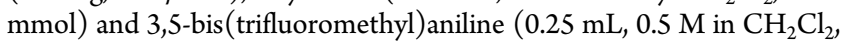
$0.12 \mathrm{mmol})$ as the nucleophile in $\mathrm{CH}_{2} \mathrm{Cl}_{2}(0.6 \mathrm{~mL})$ according to General Procedure I. Preparative TLC (c-hexane/EtOAc 5:1) yielded $38 \mathrm{mg}(48 \%)$ of the aminated product as a colorless oil. ${ }^{1} \mathbf{H}$ NMR (400 $\left.\mathrm{MHz}, \mathrm{CDCl}_{3}\right) \delta 8.09(\mathrm{dd}, J=8.4,1.2 \mathrm{~Hz}, 2 \mathrm{H}), 7.98(\mathrm{dd}, J=8.4,1.2$ $\mathrm{Hz}, 2 \mathrm{H}), 7.77-7.69(\mathrm{~m}, 2 \mathrm{H}), 7.64-7.61(\mathrm{~m}, 2 \mathrm{H}), 7.56-7.52(\mathrm{~m}, 2 \mathrm{H})$, 7.21 (br s, $1 \mathrm{H}), 7.00($ br s, $2 \mathrm{H}), 5.02(\mathrm{~s}, 1 \mathrm{H}), 4.93(\mathrm{~s}, 1 \mathrm{H}), 4.20(\mathrm{~s}$, $1 \mathrm{H}), 3.54(\mathrm{qd}, J=17.6,2.4, \mathrm{~Hz}, 1 \mathrm{H}), 3.24(\mathrm{t}, J=9.2 \mathrm{~Hz}, 1 \mathrm{H}), 2.97(\mathrm{~d}$, $J=17.6 \mathrm{~Hz}, 1 \mathrm{H}), 2.83(\mathrm{dd}, J=16.0,8.4 \mathrm{~Hz}, 1 \mathrm{H}), 2.67$ (ddd, $J=16.0$, 8.4, $1.6 \mathrm{~Hz}, 1 \mathrm{H}), 1.45$ (s, 3H), $1.38(\mathrm{~s}, 3 \mathrm{H}) \mathrm{ppm} .{ }^{13} \mathrm{C}$ NMR $(101$ $\left.\mathrm{MHz}, \mathrm{CDCl}_{3}\right) \delta 146.6,146.1,137.0,135.8,134.9,134.7,132.4(\mathrm{q}, J=$ $32.0 \mathrm{~Hz}), 131.0,130.9,128.8,128.7,123.4(\mathrm{q}, J=271.0 \mathrm{~Hz}), 114.4(\mathrm{q}$, $J=4.0 \mathrm{~Hz}$ ), 111.8, 110.8 (sept, $J=4.0 \mathrm{~Hz}$ ), 92.3, 56.7, 50.2, 41.0, 33.3, 25.9, 25.2 ppm. HRMS-ESI calcd for $\mathrm{C}_{29} \mathrm{H}_{26} \mathrm{~F}_{6} \mathrm{NO}_{4} \mathrm{~S}_{2}^{-}[\mathrm{M}-\mathrm{H}]^{-}$: 630.1213; found: 630.1215 .

$\mathrm{N}$-(2-(2-Methylene-4,4-bis(phenylsulfonyl)cyclopentyl)propan-2yl)naphthalen-1-amine (4r). Obtained using catalyst A (1.9 mg, 2.5 $\mu \mathrm{mol})$, enyne $1 \mathbf{b}\left(0.12 \mathrm{~mL}, 1.0 \mathrm{M}\right.$ in dry $\left.\mathrm{CH}_{2} \mathrm{Cl}_{2}, 0.12 \mathrm{mmol}\right)$, and naphtalen-1-amine $(18 \mathrm{mg}, 0.12 \mathrm{mmol})$ as the nucleophile in $\mathrm{CH}_{2} \mathrm{Cl}_{2}$ $(0.8 \mathrm{~mL})$ according to General Procedure I. Preparative TLC (chexane/EtOAc 5:1) yielded $50 \mathrm{mg}(74 \%)$ of the aminated product as a colorless solid. ${ }^{1} \mathrm{H}$ NMR $\left(400 \mathrm{MHz}, \mathrm{CDCl}_{3}\right) \delta 8.07(\mathrm{dd}, J=8.4,1.2$ $\mathrm{Hz}, 2 \mathrm{H}), 7.98(\mathrm{dd}, J=8.4,1.2 \mathrm{~Hz}, 2 \mathrm{H}), 7.84-7.80(\mathrm{~m}, 2 \mathrm{H}), 7.71-$ $7.66(\mathrm{~m}, 2 \mathrm{H}), 7.56-7.47(\mathrm{~m}, 6 \mathrm{H}), 7.36-7.31(\mathrm{~m}, 2 \mathrm{H}), 6.87(\mathrm{dd}, J=$ 7.2, $1.6 \mathrm{~Hz}, 1 \mathrm{H}), 5.04$ (br s, $1 \mathrm{H}), 4.98$ (br s, $1 \mathrm{H}), 4.39$ (br s, $1 \mathrm{H})$, $3.65-3.60(\mathrm{~m}, 2 \mathrm{H}), 2.91-2.85(\mathrm{~m}, 2 \mathrm{H}), 2.64(\mathrm{ddd}, J=15.6,8.8,1.6$ $\mathrm{Hz}, 1 \mathrm{H}), 1.53(\mathrm{~s}, 3 \mathrm{H}), 1.45$ (s, 3H) ppm. ${ }^{13} \mathrm{C}$ NMR $(101 \mathrm{MHz}$, $\left.\mathrm{CDCl}_{3}\right) \delta 146.7,140.9,137.2,135.8,134.7,134.6,134.5,131.0,130.9$, $128.7,128.6,125.6,125.0,124.9,119.9,118.3,111.4,109.1,91.7,56.5$, 49.7, 40.9, 33.7, 26.1, $25.4 \mathrm{ppm}$. HRMS-ESI calcd for $\mathrm{C}_{31} \mathrm{H}_{31} \mathrm{NO}_{4} \mathrm{~S}_{2} \mathrm{Na}^{+}[\mathrm{M}+\mathrm{Na}]^{+}: 568.1587$; found: 568.1591. Mp 89$92{ }^{\circ} \mathrm{C}$.

$N-(2-(2-M e t h y l e n e-4,4-b i s(p h e n y l s u l f o n y l) c y c l o p e n t y l) p r o p a n-2-$ yl)quinolin-8-amine (4s). Obtained using catalyst A (1.9 mg, 2.5 $\mu \mathrm{mol})$, enyne $1 \mathbf{b}\left(0.12 \mathrm{~mL}, 1.0 \mathrm{M}\right.$ in dry $\left.\mathrm{CH}_{2} \mathrm{Cl}_{2}, 0.12 \mathrm{mmol}\right)$, and quinolin-8-amine (20 mg, $0.12 \mathrm{mmol}$ ) as the nucleophile in $\mathrm{CH}_{2} \mathrm{Cl}_{2}$ $(0.8 \mathrm{~mL})$ according to General Procedure I. Preparative TLC (chexane/EtOAc 5:1) yielded slightly contaminated product. Recrystallization from c-hexane/EtOAc yielded $49 \mathrm{mg}(72 \%)$ of the pure aminated product as a pale yellow solid. ${ }^{1} \mathrm{H}$ NMR $\left(400 \mathrm{MHz}, \mathrm{CDCl}_{3}\right)$ $\delta 8.77$ (dd, $J=4.0,1.6 \mathrm{~Hz}, 1 \mathrm{H}$ ), 8.07 (dd, $J=8.4,2.0 \mathrm{~Hz}, 1 \mathrm{H}), 8.04$ (dd, $J=8.8,1.2 \mathrm{~Hz}, 2 \mathrm{H}), 7.98(\mathrm{dd}, J=8.8,1.2 \mathrm{~Hz}, 2 \mathrm{H}), 7.71-7.41(\mathrm{~m}$, $6 \mathrm{H}), 7.38(\mathrm{dd}, J=8.4,4.4 \mathrm{~Hz}, 1 \mathrm{H}), 7.34(\mathrm{t}, J=8.4 \mathrm{~Hz}, 1 \mathrm{H}), 7.08(\mathrm{dd}$, $J=8.0,0.8 \mathrm{~Hz}, 1 \mathrm{H}), 6.89(\mathrm{dd}, J=8.4,0.8 \mathrm{~Hz}, 1 \mathrm{H}), 6.66(\mathrm{~s}, 1 \mathrm{H}), 5.04$ (s, 1H), $4.97(\mathrm{~s}, 1 \mathrm{H}), 3.69-3.59(\mathrm{~m}, 2 \mathrm{H}), 2.86-2.77(\mathrm{~m}, 2 \mathrm{H}), 2.53$ (ddd, $J=15.6,8.8,1.6 \mathrm{~Hz}), 1.57(\mathrm{~s}, 3 \mathrm{H}), 1.45(\mathrm{~s}, 3 \mathrm{H}) \mathrm{ppm} .{ }^{13} \mathrm{C}$ NMR $\left(101 \mathrm{MHz}, \mathrm{CDCl}_{3}\right) \delta 146.8,146.7,142.0,138.6,137.0,136.2,135.6$, $134.7,134.5,131.0,130.9,130.8,129.6,129.1,128.9,128.7,128.6$, $128.5,127.5,121.4,114.3,111.2,107.2,91.6,55.9,48.4,40.8,33.6$, 25.8, 25.0 ppm. HRMS-ESI calcd for $\mathrm{C}_{30} \mathrm{H}_{31} \mathrm{~N}_{2} \mathrm{O}_{4} \mathrm{~S}_{2}{ }^{+}[\mathrm{M}+\mathrm{H}]^{+}$: 547.1720; found: 547.1720. $\mathrm{Mp} 82-85^{\circ} \mathrm{C}$.

$\mathrm{N}$-Methyl-N-(2-(2-methylene-4,4-bis(phenylsulfonyl)cyclopentyl)propan-2-yl)aniline (4t). Obtained using catalyst A (1.9 mg, 2.5 $\mu \mathrm{mol})$, enyne $1 \mathbf{b}\left(0.12 \mathrm{~mL}, 1.0 \mathrm{M}\right.$ in dry $\left.\mathrm{CH}_{2} \mathrm{Cl}_{2}, 0.12 \mathrm{mmol}\right)$, and $\mathrm{N}$ methylaniline $\left(0.25 \mathrm{~mL}, 0.5 \mathrm{M}\right.$ in $\left.\mathrm{CH}_{2} \mathrm{Cl}_{2}, 0.12 \mathrm{mmol}\right)$ as the nucleophile in $\mathrm{CH}_{2} \mathrm{Cl}_{2}(0.6 \mathrm{~mL})$ according to General Procedure I. Preparative TLC (c-hexane/EtOAc 5:1) yielded $53 \mathrm{mg}$ (83\%) of the aminated product as a colorless solid. ${ }^{1} \mathbf{H}$ NMR $\left(400 \mathrm{MHz}, \mathrm{CDCl}_{3}\right) \delta$ $8.15(\mathrm{dd}, J=7.6,1.2 \mathrm{~Hz}, 2 \mathrm{H}), 8.07(\mathrm{dd}, J=7.6,1.2 \mathrm{~Hz}, 2 \mathrm{H}), 7.77-$ $7.71(\mathrm{~m}, 2 \mathrm{H}), 7.66-7.58(\mathrm{~m}, 4 \mathrm{H}), 7.33-7.30(\mathrm{~m}, 2 \mathrm{H}), 7.17-7.14(\mathrm{~m}$, $3 \mathrm{H}), 5.12(\mathrm{~s}, 1 \mathrm{H}), 5.05(\mathrm{~s}, 1 \mathrm{H}), 3.56(\mathrm{qd}, J=17.2,2.0 \mathrm{~Hz}, 1 \mathrm{H}), 3.22$ $(\mathrm{t}, J=7.6 \mathrm{~Hz}, 1 \mathrm{H}), 3.08(\mathrm{dd}, J=15.6,8.0 \mathrm{~Hz}, 1 \mathrm{H}), 2.80(\mathrm{~d}, J=17.2$ $\mathrm{Hz}, 1 \mathrm{H}), 2.72-2.69(\mathrm{~m}, 1 \mathrm{H}+3 \mathrm{H}), 1.18(\mathrm{~s}, 3 \mathrm{H}), 0.85(\mathrm{~s}, 3 \mathrm{H}) \mathrm{ppm} .{ }^{13} \mathrm{C}$ NMR $\left(101 \mathrm{MHz}, \mathrm{CDCl}_{3}\right) \delta 150.5,147.3,137.3,135.9,134.6,134.5$, 131.1, 131.0, 128.7, 128.6, 128.2, 128.1, 124.4, 111.3, 91.9, 60.4, 50.4, 40.9, 36.4, 34.0, 21.8, $20.7 \mathrm{ppm}$. HRMS-ESI calcd for $\mathrm{C}_{28} \mathrm{H}_{31} \mathrm{NO}_{4} \mathrm{~S}_{2} \mathrm{Na}^{+}[\mathrm{M}+\mathrm{Na}]^{+}$: 532.1587; found: 532.1586. Mp 171$172{ }^{\circ} \mathrm{C}$.

Dimethyl 3-Methylene-4-(2-(phenylamino)propan-2-yl)cyclopentane-1,1-dicarboxylate (4u). Obtained using catalyst A (2.2 mg, $2.8 \mu \mathrm{mol})$, enyne $1 \mathrm{c}(35 \mathrm{mg}, 0.15 \mathrm{mmol})$, and aniline $(15 \mu \mathrm{L}, 0.16$ mol) as the nucleophile in $\mathrm{CH}_{2} \mathrm{Cl}_{2}(1.0 \mathrm{~mL})$ according to General Procedure I. Purification by column chromatography (c-hexane/ EtOAc 13:1) yielded $40 \mathrm{mg}(83 \%)$ of the aminated product as a colorless oil. ${ }^{1} \mathrm{H}$ NMR (400 MHz, $\left.\mathrm{CDCl}_{3}\right) \delta 7.17-7.11(\mathrm{~m}, 2 \mathrm{H})$, 6.76-6.69 (m, 3H), 5.07 (br s, 1H), 4.97 (br s, 1H), $3.72(\mathrm{~s}, 3 \mathrm{H}), 3.70$ (s, 3H), 3.61 (br s, $1 \mathrm{H}), 3.23-3.19(\mathrm{~m}, 1 \mathrm{H}), 2.94-2.84(\mathrm{~m}, 2 \mathrm{H}), 2.63$ (ddd, $J=13.5,8.4,1.2 \mathrm{~Hz}, 1 \mathrm{H}), 2.05(\mathrm{dd}, J=13.5,9.4 \mathrm{~Hz}, 1 \mathrm{H}), 1.31$ (s, 3H), 1.29 (s, 3H) ppm. ${ }^{13} \mathrm{C}$ NMR $\left(101 \mathrm{MHz}, \mathrm{CDCl}_{3}\right) \delta 172.0$, $171.9,148.5,146.3,129.2,118.3,117.2,111.1,58.6,56.3,52.8,49.1$, 44.0, 36.4, 27.0, 25.9, 25.8 ppm. HRMS-ESI calcd for $\mathrm{C}_{19} \mathrm{H}_{26} \mathrm{NO}_{4}^{+}[\mathrm{M}$ $+\mathrm{H}]^{+}:$332.1856; found: 332.1857.

Dimethyl 3-(2-((4-Methoxyphenyl)amino)propan-2-yl)-4methylenecyclopentane-1,1-dicarboxylate (4v). Obtained using catalyst A (2.3 mg, $3.0 \mu \mathrm{mol})$, enyne $1 \mathrm{c}(36 \mathrm{mg}, 0.15 \mathrm{mmol})$, and p-anisidine $(20 \mathrm{mg}, 0.16 \mathrm{mmol})$ as the nucleophile in $\mathrm{CH}_{2} \mathrm{Cl}_{2}(1 \mathrm{~mL})$ according to General Procedure I. Purification by column chromatography (c-hexane/EtOAc $8: 1$ ) yielded $44 \mathrm{mg}(81 \%)$ of the aminated product as a yellow oil. ${ }^{1} \mathrm{H}$ NMR $\left(400 \mathrm{MHz}, \mathrm{CDCl}_{3}\right) \delta 6.75(\mathrm{br} \mathrm{s}$, 4H), 5.10 (br s, 1H), 5.04 (br s, $1 \mathrm{H}), 3.75$ (s, 3H), $3.73(\mathrm{~s}, 3 \mathrm{H}), 3.71$ (s, 3H), $3.16($ br s, $1 \mathrm{H}), 3.03-2.83(\mathrm{~m}, 3 \mathrm{H}), 2.64$ (ddd, $J=13.4,8.5$, $1.7 \mathrm{~Hz}, 1 \mathrm{H}), 2.12(\mathrm{dd}, J=13.4,9.3 \mathrm{~Hz}, 1 \mathrm{H}), 1.21(\mathrm{~s}, 3 \mathrm{H}), 1.19(\mathrm{~s}, 3 \mathrm{H})$ ppm. ${ }^{13} \mathrm{C}$ NMR $\left(101 \mathrm{MHz}, \mathrm{CDCl}_{3}\right) \delta 172.1,172.0,154.0,148.7$, $139.4,122.0,114.4,111.3,58.7,56.9,55.6,52.8,50.4,44.0,36.4,27.0$, 
25.9, 25.7 ppm. HRMS-ESI calcd for $\mathrm{C}_{20} \mathrm{H}_{27} \mathrm{NO}_{5} \mathrm{Na}^{+}[\mathrm{M}+\mathrm{Na}]^{+}$: 384.1781; found: 384.1801 .

Dimethyl 3-(2-((2-Bromophenyl)amino)propan-2-yl)-4methylenecyclopentane-1,1-dicarboxylate (4w). Obtained using catalyst A (34 mg, $0.04 \mathrm{mmol})$, enyne $1 \mathrm{c}(525 \mathrm{mg}, 2.20 \mathrm{mmol})$, and 2-bromoaniline (417 $\mathrm{mg}, 2.42 \mathrm{mmol}$ ) as the nucleophile in $\mathrm{CH}_{2} \mathrm{Cl}_{2}(14 \mathrm{~mL})$ according to General Procedure I. Purification by column chromatography (pentane $/ \mathrm{Et}_{2} \mathrm{O} 9: 1$ ) yielded $649 \mathrm{mg}(72 \%)$ of the aminated product as a colorless oil. ${ }^{1} \mathbf{H}$ NMR $(500 \mathrm{MHz}$, $\left.\mathrm{CDCl}_{3}\right) \delta 7.43(\mathrm{dd}, J=7.9,1.5 \mathrm{~Hz}, 1 \mathrm{H}), 7.12(\mathrm{ddd}, J=8.6,7.4,1.6 \mathrm{~Hz}$, $1 \mathrm{H}), 6.95$ (dd, $J=8.3,1.5 \mathrm{~Hz}, 1 \mathrm{H}$ ), 6.55 (ddd, $J=7.9,7.3,1.5 \mathrm{~Hz}$, $1 \mathrm{H}), 5.08(\mathrm{t}, J=2.3 \mathrm{~Hz}, 1 \mathrm{H}), 4.91(\mathrm{ddt}, J=2.9,1.8,0.9 \mathrm{~Hz}, 1 \mathrm{H}), 4.43$ $(\mathrm{s}, 1 \mathrm{H}), 3.73(\mathrm{~s}, 3 \mathrm{H}), 3.71(\mathrm{~s}, 3 \mathrm{H}), 3.27$ (ddd, $J=9.4,8.5,2.0 \mathrm{~Hz}, 1 \mathrm{H})$, $2.95(\mathrm{dq}, J=15.3,2.6 \mathrm{~Hz}, 1 \mathrm{H}), 2.88(\mathrm{ddt}, J=15.2,1.9,1.1 \mathrm{~Hz}, 1 \mathrm{H})$, 2.62 (ddd, $J=13.6,8.6,1.9 \mathrm{~Hz}, 1 \mathrm{H}), 2.02(\mathrm{dd}, J=13.6,9.5 \mathrm{~Hz}, 1 \mathrm{H})$, $1.38(\mathrm{~s}, 3 \mathrm{H}), 1.36(\mathrm{~s}, 3 \mathrm{H}) \mathrm{ppm} .{ }^{13} \mathrm{C}$ NMR $\left(126 \mathrm{MHz} \mathrm{CDCl}_{3}\right) \delta$ $172.0,171.9,147.9,143.3,133.0,128.2,118.1,114.8,112.1,111.5$, 58.7, 56.7, 52.9, 48.8, 44.1, 36.4, 25.7, 25.6 ppm. HRMS-ESI calcd for $\mathrm{C}_{19} \mathrm{H}_{24} \mathrm{BrNO}_{4} \mathrm{Na}^{+}[\mathrm{M}+\mathrm{Na}]^{+}: 432.0781$; found: 432.0785 .

Dimethyl 3-(2-((4-Bromophenyl)amino)propan-2-yl)-4methylenecyclopentane-1,1-dicarboxylate (4x). Obtained using catalyst A (2.3 mg, $3.0 \mu \mathrm{mol})$, enyne $1 \mathrm{c}(35 \mathrm{mg}, 0.15 \mathrm{mmol})$, and 4-bromoaniline ( $28 \mathrm{mg}, 0.16 \mathrm{mmol})$ as the nucleophile in $\mathrm{CH}_{2} \mathrm{Cl}_{2}(1.0$ $\mathrm{mL}$ ) according to General Procedure I. Purification by column chromatography (c-hexane/EtOAc 9:1) yielded $37 \mathrm{mg}$ (62\%) of the aminated product as a colorless solid. ${ }^{1} \mathbf{H}$ NMR $\left(400 \mathrm{MHz}, \mathrm{CDCl}_{3}\right) \delta$ 7.27-7.24 (m, 2H), 6.65-6.58 (m, 2H), 5.09 (br s, 1H), 4.95 (br s, $1 \mathrm{H}), 3.75(\mathrm{~s}, 3 \mathrm{H}), 3.73(\mathrm{~s}, 3 \mathrm{H}), 3.69(\mathrm{br} \mathrm{s}, 1 \mathrm{H}), 3.23-3.17(\mathrm{~m}, 1 \mathrm{H})$, 2.90 (br s, $2 \mathrm{H}), 2.62(\mathrm{dd}, J=13.5,8.4 \mathrm{~Hz}, 1 \mathrm{H}), 2.03$ (dd, $J=13.5,9.3$ $\mathrm{Hz}, 1 \mathrm{H}), 1.32$ (s, 3H), 1.29 (s, 3H) ppm. ${ }^{13} \mathrm{C}$ NMR (101 MHz, $\left.\mathrm{CDCl}_{3}\right) \delta 172.0,171.8,148.3,145.4,132.0,118.4,111.2,110.1,58.6$, 56.4, 52.9, 48.9, 44.0, 36.4, $25.8 \mathrm{ppm}$. HRMS-ESI calcd for $\mathrm{C}_{19} \mathrm{H}_{25} \mathrm{BrNO}_{4}{ }^{+}\left[\mathrm{M}+\mathrm{H}^{+}:\right.$: 410.0961; found: 410.0947. Mp 116-118 ${ }^{\circ} \mathrm{C}$.

Dimethyl 3-(2-((2-lodophenyl)amino)propan-2-yl)-4-methylenecyclopentane-1,1-dicarboxylate (4y). Obtained using catalyst A (34.2 $\mathrm{mg}, 0.04 \mathrm{mmol})$, enyne $1 \mathrm{c}(500 \mathrm{mg}, 2.1 \mathrm{mmol})$, and 2iodoaniline (492 mg, $2.25 \mathrm{mmol}$ ) as the nucleophile in $\mathrm{CH}_{2} \mathrm{Cl}_{2}(6.0$ $\mathrm{mL}$ ) according to General Procedure I. Purification by column chromatography (pentane/ $\mathrm{Et}_{2} \mathrm{O} 10: 1$ to $4: 1$ ) yielded $553 \mathrm{mg}$ (58\%) of the aminated product as a colorless oil. ${ }^{1} \mathbf{H}$ NMR $\left(500 \mathrm{MHz}, \mathrm{CDCl}_{3}\right)$ $\delta 7.68(\mathrm{dd}, J=7.9,1.6 \mathrm{~Hz}, 1 \mathrm{H}), 7.16(\mathrm{ddd}, J=8.5,7.2,1.6 \mathrm{~Hz}, 1 \mathrm{H})$, $6.91(\mathrm{dd}, J=8.4,1.5 \mathrm{~Hz}, 1 \mathrm{H}), 6.42(\mathrm{ddd}, J=8.0,7.3,1.4 \mathrm{~Hz}, 1 \mathrm{H}), 5.10$ (br s, $1 \mathrm{H}), 4.92(\mathrm{br} \mathrm{s}, 1 \mathrm{H}), 4.25(\mathrm{~s}, 1 \mathrm{H}), 3.74(\mathrm{~s}, 3 \mathrm{H}), 3.72(\mathrm{~s}, 3 \mathrm{H})$, $3.31-3.25(\mathrm{~m}, 1 \mathrm{H}), 2.97(\mathrm{dq}, J=15.2,2.6 \mathrm{~Hz}, 1 \mathrm{H}), 2.90(\mathrm{~d}, J=15.3$ $\mathrm{Hz}, 1 \mathrm{H}), 2.63$ (ddd, $J=13.7,8.6,2.0 \mathrm{~Hz}, 1 \mathrm{H}), 2.04(\mathrm{dd}, J=13.6,9.5$ $\mathrm{Hz}, 1 \mathrm{H}), 1.40(\mathrm{~s}, 3 \mathrm{H}), 1.36(\mathrm{~s}, 3 \mathrm{H}) \mathrm{ppm} .{ }^{13} \mathrm{C}$ NMR $(126 \mathrm{MHz}$, $\left.\mathrm{CDCl}_{3}\right) \delta 171.7,171.7,147.7,145.4,139.6,129.0,118.8,113.8,111.4$, 88.7, 58.5, 56.9, 52.7, 48.6, 43.9, 36.2, 25.6, 25.3 ppm. HRMS-ESI calcd for $\mathrm{C}_{19} \mathrm{H}_{24} \mathrm{INO}_{4} \mathrm{Na}^{+}[\mathrm{M}+\mathrm{Na}]^{+}$: 480.0642 , found 480.0638 .

Dimethyl 3-(2-((4-Acetylphenyl)amino)propan-2-yl)-4methylenecyclopentane-1,1-dicarboxylate (4z). Obtained using catalyst A $(2.3 \mathrm{mg}, 2.9 \mu \mathrm{mol})$, enyne $1 \mathrm{c}(0.15 \mathrm{~mL}, 1.0 \mathrm{M}$ in dry $\mathrm{CH}_{2} \mathrm{Cl}_{2}, 0.15 \mathrm{mmol}$ ), and 1-(4-aminophenyl)ethanone $(20 \mathrm{mg}, 0.15$ mmol $)$ as the nucleophile in $\mathrm{CH}_{2} \mathrm{Cl}_{2}(0.8 \mathrm{~mL})$ according to General Procedure I. Preparative TLC (c-hexane/EtOAc 5:1) yielded $40 \mathrm{mg}$ (74\%) of the aminated product as a colorless oil. ${ }^{1} \mathrm{H}$ NMR $(400 \mathrm{MHz}$, $\left.\mathrm{CDCl}_{3}\right) \delta 7.87-7.85(\mathrm{~m}, 2 \mathrm{H}), 6.69-6.66(\mathrm{~m}, 2 \mathrm{H}), 5.09(\mathrm{~s}, 1 \mathrm{H}), 4.91$ $(\mathrm{s}, 1 \mathrm{H}), 4.31(\mathrm{~s}, 1 \mathrm{H}), 3.75(\mathrm{~s}, 3 \mathrm{H}), 3.73(\mathrm{~s}, 3 \mathrm{H}), 3.37(\mathrm{t}, J=9.2 \mathrm{~Hz}$, $1 \mathrm{H}), 2.92(\mathrm{~s}, 2 \mathrm{H}), 2.65(\mathrm{dd}, J=13.6,8.8 \mathrm{~Hz}, 1 \mathrm{H}), 2.51(\mathrm{~s}, 3 \mathrm{H}), 2.00$ (dd, $J=13.6,8.8 \mathrm{~Hz}, 1 \mathrm{H}), 1.41(\mathrm{~s}, 3 \mathrm{H}), 1.39(\mathrm{~s}, 3 \mathrm{H}) \mathrm{ppm} .{ }^{13} \mathrm{C}$ NMR $\left(101 \mathrm{MHz}, \mathrm{CDCl}_{3}\right) \delta 196.2,171.8,171.6,150.5,147.9,130.7,126.6$, 113.7, 111.0, 58.4, 56.3, 52.8, 48.0, 43.9, 36.2, 26.0, 25.5, 25.4 ppm. HRMS-ESI calcd for $\mathrm{C}_{21} \mathrm{H}_{26} \mathrm{NO}_{5}^{-}[\mathrm{M}-\mathrm{H}]^{-}:$372.1816; found: 372.1817.

Dimethyl 3-(2-((3,5-Bis(trifluoromethyl)phenyl)amino)propan-2yl)-4-methylenecyclopentane-1,1-dicarboxylate (4aa). Obtained using catalyst A $(2.4 \mathrm{mg}, 3 \mu \mathrm{mol})$, enyne $1 \mathrm{c}(35 \mathrm{mg}, 0.14 \mathrm{mmol})$, and 3,5-bis(trifluoromethyl)aniline $(25 \mu \mathrm{L}, 0.16 \mathrm{mmol})$ as the nucleophile in $\mathrm{CH}_{2} \mathrm{Cl}_{2}(1.0 \mathrm{~mL})$ according to General Procedure I.
Preparative TLC (c-hexane/EtOAc 15:1) yielded $28 \mathrm{mg}$ (42\%) of the aminated product as a colorless oil. ${ }^{1} \mathbf{H}$ NMR $\left(400 \mathrm{MHz}, \mathrm{CDCl}_{3}\right) \delta$ $7.16(\mathrm{~s}, 1 \mathrm{H}), 7.04(\mathrm{~s}, 2 \mathrm{H}), 5.15-5.12(\mathrm{~m}, 1 \mathrm{H}), 4.99-4.96(\mathrm{~m}, 1 \mathrm{H})$, 4.17 (br s, $1 \mathrm{H}), 3.74(\mathrm{~s}, 3 \mathrm{H}), 3.72(\mathrm{~s}, 3 \mathrm{H}), 3.18$ (dddd, $J=10.3$, 8.6, $4.0,2.0 \mathrm{~Hz}, 1 \mathrm{H}), 2.94(\mathrm{dq}, J=15.3,1.2 \mathrm{~Hz}, 1 \mathrm{H}), 2.88(\mathrm{dq}, J=15.4,2.6$ $\mathrm{Hz}, 1 \mathrm{H}), 2.62$ (ddd, $J=13.6,8.5,1.8 \mathrm{~Hz}, 1 \mathrm{H}), 2.04$ (dd, $J=13.6,9.6$ $\mathrm{Hz}, 1 \mathrm{H}), 1.40(\mathrm{~s}, 3 \mathrm{H}), 1.36$ (s, 3H) ppm. ${ }^{13} \mathrm{C}$ NMR (101 MHz, $\left.\mathrm{CDCl}_{3}\right) \delta 171.7,171.5,147.8,147.0,132.3(\mathrm{q}, J=32.7 \mathrm{~Hz}), 123.5(\mathrm{q}, J$ $=272.7 \mathrm{~Hz}$ ), 114.6 (app. d, $J=4.2 \mathrm{~Hz}$ ), 111.4, 110.5 (quintet, $J=3.7$ $\mathrm{Hz}), 58.3,56.5,52.8,52.8,49.0,43.8,36.1,25.6,25.3 \mathrm{ppm} .{ }^{19} \mathrm{~F}$ NMR $\left(376 \mathrm{MHz}, \mathrm{CDCl}_{3}\right) \delta-63.3 \mathrm{ppm}$. HRMS-ESI calcd for $\mathrm{C}_{21} \mathrm{H}_{23} \mathrm{~F}_{6} \mathrm{NO}_{4} \mathrm{Na}^{+}[\mathrm{M}+\mathrm{Na}]^{+}$: 490.1423; found: 490.1430 .

Dimethyl 3-Methylene-4-(2-(naphthalen-1-ylamino)propan-2yl)cyclopentane-1,1-dicarboxylate (4ab). Obtained using catalyst A $(2.3 \mathrm{mg}, 2.9 \mu \mathrm{mol})$, enyne $1 \mathrm{c}\left(0.15 \mathrm{~mL}, 1.0 \mathrm{M}\right.$ in dry $\mathrm{CH}_{2} \mathrm{Cl}_{2}, 0.15$ $\mathrm{mmol}$ ), and naphtalen-1-amine $(21 \mathrm{mg}, 0.15 \mathrm{mmol})$ as the nucleophile in $\mathrm{CH}_{2} \mathrm{Cl}_{2}(0.8 \mathrm{~mL})$ according to General Procedure I. Preparative TLC (c-hexane/EtOAc 5:1) yielded $44 \mathrm{mg}(78 \%)$ of the aminated product as a colorless oil. ${ }^{1} \mathbf{H}$ NMR $\left(400 \mathrm{MHz}, \mathrm{CDCl}_{3}\right) \delta 7.82-7.78$ $(\mathrm{m}, 2 \mathrm{H}), 7.48-7.43(\mathrm{~m}, 2 \mathrm{H}), 7.34(\mathrm{t}, J=8.0 \mathrm{~Hz}, 1 \mathrm{H}), 7.26(\mathrm{~d}, J=8.0$ $\mathrm{Hz}, 1 \mathrm{H}), 6.97(\mathrm{~d}, J=7.6 \mathrm{~Hz}, 1 \mathrm{H}), 5.10(\mathrm{br} \mathrm{s}, 1 \mathrm{H}), 4.94($ br s, $1 \mathrm{H})$, $4.41(\mathrm{~s}, 1 \mathrm{H}), 3.75(\mathrm{~s}, 3 \mathrm{H}), 3.74(\mathrm{~s}, 3 \mathrm{H}), 3.48(\mathrm{dt}, J=9.2,1.6 \mathrm{~Hz}, 1 \mathrm{H})$, $3.03-2.92(\mathrm{~m}, 2 \mathrm{H}), 2.72(\mathrm{ddd}, J=13.6,8.8,1.6 \mathrm{~Hz}, 1 \mathrm{H}), 2.14(\mathrm{dd}, J=$ 13.6, $8.8 \mathrm{~Hz}, 1 \mathrm{H}), 1.47(\mathrm{~s}, 3 \mathrm{H}), 1.46(\mathrm{~s}, 3 \mathrm{H}) \mathrm{ppm} .{ }^{13} \mathrm{C}$ NMR $(101$ $\left.\mathrm{MHz}, \mathrm{CDCl}_{3}\right) \delta 171.9,171.8,148.3,140.9,134.7,128.9,126.2,125.5$, $125.1,124.8,120.1,117.9,111.2,109.3,58.6,55.5,52.8,48.7,44.0$, 36.4, 25.5 ppm. HRMS-ESI calcd for $\mathrm{C}_{23} \mathrm{H}_{28} \mathrm{NO}_{4}{ }^{+}[\mathrm{M}+\mathrm{H}]^{+}$: 382.2013; found: 382.2022 .

Dimethyl 3-Methylene-4-(2-(quinolin-8-ylamino)propan-2-yl)cyclopentane-1,1-dicarboxylate (4ac). Obtained using catalyst A $(2.3 \mathrm{mg}, 2.94 \mu \mathrm{mol})$, enyne $1 \mathrm{c}\left(0.15 \mathrm{~mL}, 1.0 \mathrm{M}\right.$ in dry $\mathrm{CH}_{2} \mathrm{Cl}_{2}, 0.15$ $\mathrm{mmol})$, and quinolin-8-amine $(21 \mathrm{mg}, 0.15 \mathrm{mmol})$ as the nucleophile in $\mathrm{CH}_{2} \mathrm{Cl}_{2}(0.8 \mathrm{~mL})$ according to General Procedure I. Preparative TLC (c-hexane/EtOAc 5:1) yielded $45 \mathrm{mg}$ (81\%) of the aminated product as a pale yellow oil. ${ }^{1} \mathrm{H}$ NMR $\left(400 \mathrm{MHz}, \mathrm{CDCl}_{3}\right) \delta 8.71(\mathrm{dd}, J$ $=4.0,1.6 \mathrm{~Hz}, 1 \mathrm{H}), 8.05(\mathrm{dd}, J=8.4,1.6 \mathrm{~Hz}, 1 \mathrm{H}), 7.38-7.33(\mathrm{~m}, 2 \mathrm{H})$, $7.05(\mathrm{~d}, J=8.0 \mathrm{~Hz}, 1 \mathrm{H}), 6.98(\mathrm{~d}, J=8.4 \mathrm{~Hz}, 1 \mathrm{H}), 6.65(\mathrm{~s}, 1 \mathrm{H}), 5.05$ $(\mathrm{s}, 1 \mathrm{H}), 4.89(\mathrm{~s}, 1 \mathrm{H}), 3.73(\mathrm{~s}, 3 \mathrm{H}), 3.72(\mathrm{~s}, 3 \mathrm{H}), 3.59-3.55(\mathrm{~m}, 1 \mathrm{H})$, $3.01(\mathrm{qd}, J=15.2,2.0 \mathrm{~Hz}, 1 \mathrm{H}), 2.92(\mathrm{~d}, J=15.2 \mathrm{~Hz}, 1 \mathrm{H}), 2.66(\mathrm{ddd}, J$ $=13.6,8.4,1.6 \mathrm{~Hz}, 1 \mathrm{H}), 2.07(\mathrm{dd}, J=13.6,8.4 \mathrm{~Hz}, 1 \mathrm{H}), 1.57(\mathrm{~s}, 3 \mathrm{H})$, $1.53(\mathrm{~s}, 3 \mathrm{H}) \mathrm{ppm} .{ }^{13} \mathrm{C}$ NMR $\left(101 \mathrm{MHz}, \mathrm{CDCl}_{3}\right) \delta 171.9,171.8,148.1$, $146.6,142.3,138.7,136.1,128.9,127.5,121.2,113.5,110.9,106.9$, 58.6, 55.6, 52.7, 47.5, 44.0, 36.3, 30.9, 25.2, 25.0 ppm. HRMS-ESI calcd for $\mathrm{C}_{22} \mathrm{H}_{27} \mathrm{~N}_{2} \mathrm{O}_{4}^{+}[\mathrm{M}+\mathrm{H}]^{+}$: 383.1965; found: 383.1975 .

Dimethyl 3-(2-(Methyl(phenyl)amino)propan-2-yl)-4-methylenecyclopentane-1,1-dicarboxylate (4ad). Obtained using catalyst A $(3.2 \mathrm{mg}, 4.2 \mu \mathrm{mol})$, enyne $1 \mathrm{c}\left(0.21 \mathrm{~mL}, 1.0 \mathrm{M}\right.$ in dry $\mathrm{CH}_{2} \mathrm{Cl}_{2}, 0.21$ $\mathrm{mmol})$, and $\mathrm{N}$-methylaniline $\left(0.46 \mathrm{~mL}, 0.5 \mathrm{M}\right.$ in $\left.\mathrm{CH}_{2} \mathrm{Cl}_{2}, 0.23 \mathrm{mmol}\right)$ as the nucleophile in $\mathrm{CH}_{2} \mathrm{Cl}_{2}(0.5 \mathrm{~mL})$ according to General Procedure I. Preparative TLC (c-hexane/EtOAc 5:1) yielded $61 \mathrm{mg}$ $(85 \%)$ of the aminated product as a colorless oil. ${ }^{1} \mathbf{H}$ NMR $(400 \mathrm{MHz}$, $\left.\mathrm{CDCl}_{3}\right) \delta 7.25-7.23(\mathrm{~m}, 2 \mathrm{H}), 7.19-7.14(\mathrm{~m}, 2 \mathrm{H}), 7.13-7.08(\mathrm{~m}$, $1 \mathrm{H}), 5.14$ (br s, $1 \mathrm{H}), 5.08$ (br s, 1H), $3.75(\mathrm{~s}, 3 \mathrm{H}), 3.73(\mathrm{~s}, 3 \mathrm{H}), 3.10$ $3.05(\mathrm{~m}, 1 \mathrm{H}), 2.97-2.84(\mathrm{~m}, 2 \mathrm{H}), 2.73(\mathrm{~s}, 3 \mathrm{H}), 2.63$ (ddd, $J=13.8$, $8.6,1.7 \mathrm{~Hz}, 1 \mathrm{H}), 2.21(\mathrm{dd}, J=13.8,9.3 \mathrm{~Hz}, 1 \mathrm{H}), 1.08(\mathrm{~s}, 3 \mathrm{H}), 0.89(\mathrm{~s}$, $3 \mathrm{H}) \mathrm{ppm} .{ }^{13} \mathrm{C}$ NMR $\left(101 \mathrm{MHz}, \mathrm{CDCl}_{3}\right) \delta 171.9,171.8,150.9,148.9$, 128.3, 128.0, 124.1, 110.8, 60.0, 58.4, 52.7, 48.8, 44.0, 36.6, 36.3, 21.5, $20.8 \mathrm{ppm}$. HRMS-ESI calcd for $\mathrm{C}_{20} \mathrm{H}_{28} \mathrm{NO}_{4}{ }^{+}[\mathrm{M}+\mathrm{H}]^{+}:$346.2013; found: 346.2015 .

Dimethyl 3-(2-(Allyl(phenyl)amino)propan-2-yl)-4-methylenecyclopentane-1,1-dicarboxylate (4ae). Obtained using catalyst A (11 mg, $15.0 \mu \mathrm{mol})$, enyne $1 \mathrm{c}\left(0.29 \mathrm{~mL}, 1.0 \mathrm{M}\right.$ in dry $\mathrm{CH}_{2} \mathrm{Cl}_{2}, 0.29$ $\mathrm{mmol})$, and $\mathrm{N}$-allylaniline $(70 \mathrm{mg}, 0.29 \mathrm{mmol})$ as the nucleophile in $\mathrm{CH}_{2} \mathrm{Cl}_{2}(1.5 \mathrm{~mL})$ according to General Procedure I. Flash column chromatography (c-hexane/EtOAc 5:1) yielded $54 \mathrm{mg}$ (49\%) of the aminated product as a colorless solid. ${ }^{1} \mathbf{H}$ NMR $\left(400 \mathrm{MHz}, \mathrm{CDCl}_{3}\right) \delta$ $7.25(\mathrm{t}, J=8.0 \mathrm{~Hz}, 2 \mathrm{H}), 7.16(\mathrm{~d}, J=8.0 \mathrm{~Hz}, 2 \mathrm{H}), 7.11(\mathrm{t}, J=8.0 \mathrm{~Hz}$, $1 \mathrm{H}), 5.66-5.58(\mathrm{~m}, 1 \mathrm{H}), 5.16(\mathrm{~s}, 1 \mathrm{H}), 5.11(\mathrm{~s}, 1 \mathrm{H}), 4.98(\mathrm{dd}, J=17.5$, $1.5 \mathrm{~Hz}, 1 \mathrm{H}), 4.85(\mathrm{dd}, J=17.5,1.5 \mathrm{~Hz}, 1 \mathrm{H}), 3.79(\mathrm{~s}, 3 \mathrm{H}), 3.77-3.76$ $(\mathrm{m}, 3 \mathrm{H}+2 \mathrm{H}), 3.13-3.07(\mathrm{~m}, 1 \mathrm{H}), 2.96-2.87(\mathrm{~m}, 2 \mathrm{H}), 2.66(\mathrm{ddd}, J=$ 
14.0, 8.5, $1.5 \mathrm{~Hz}, 1 \mathrm{H}), 2.30$ (dd, $J=13.5,9.0 \mathrm{~Hz}, 1 \mathrm{H}), 1.11(\mathrm{~s}, 3 \mathrm{H})$, $0.88(\mathrm{~s}, 3 \mathrm{H}) \mathrm{ppm} .{ }^{13} \mathrm{C}$ NMR $\left(101 \mathrm{MHz}, \mathrm{CDCl}_{3}\right) \delta 172.2,172.1,149.1$, $147.9,137.2,130.0,127.9,124.8,115.7,110.9,60.4,58.5,52.7,52.6$, 50.6, 49.2, 44.1, 36.7, 22.2, 21.9 ppm. HRMS-ESI calcd for $\mathrm{C}_{22} \mathrm{H}_{30} \mathrm{NO}_{4}{ }^{+}[\mathrm{M}+\mathrm{H}]^{+}: 372.2169$; found: 372.2156 . Mp $75-77{ }^{\circ} \mathrm{C}$.

Dimethyl 3-(2-(Indolin-1-yl)propan-2-yl)-4-methylenecyclopentane-1,1-dicarboxylate (4af). Obtained using catalyst A (11 mg, $15.0 \mu \mathrm{mol})$, enyne $1 \mathrm{c}\left(0.29 \mathrm{~mL}, 1.0 \mathrm{M}\right.$ in dry $\left.\mathrm{CH}_{2} \mathrm{Cl}_{2}, 0.29 \mathrm{mmol}\right)$, and indoline $\left(0.29 \mathrm{~mL}, 1.0 \mathrm{M}\right.$ in dry $\left.\mathrm{CH}_{2} \mathrm{Cl}_{2}, 0.29 \mathrm{mmol}\right)$ as the nucleophile in $\mathrm{CH}_{2} \mathrm{Cl}_{2}(1.5 \mathrm{~mL})$ according to General Procedure I. Flash column chromatography (c-hexane/EtOAc 5:1) yielded $92 \mathrm{mg}$ $(87 \%)$ of the aminated product as a colorless oil. ${ }^{1} \mathbf{H}$ NMR $(400 \mathrm{MHz}$, $\left.\mathrm{CDCl}_{3}\right) \delta 7.06(\mathrm{dd}, J=7.5,0.5 \mathrm{~Hz}, 1 \mathrm{H}), 7.01(\mathrm{t}, J=8.0 \mathrm{~Hz}, 1 \mathrm{H}), 6.80$ $(\mathrm{d}, J=8.0 \mathrm{~Hz}, 1 \mathrm{H}), 6.63(\mathrm{dd}, J=7.5,0.5 \mathrm{~Hz}, 1 \mathrm{H}), 5.07($ br s, $2 \mathrm{H})$, $3.76(\mathrm{~s}, 3 \mathrm{H}), 3.75(\mathrm{~s}, 3 \mathrm{H}), 3.60(\mathrm{t}, J=10.0 \mathrm{~Hz}, 1 \mathrm{H}), 3.52-3.41(\mathrm{~m}$, $2 \mathrm{H}), 2.93(\mathrm{br} \mathrm{s}, 2 \mathrm{H}), 2.88(\mathrm{t}, J=8.5 \mathrm{~Hz}, 2 \mathrm{H}), 2.63(\mathrm{ddd}, J=13.5,8.5$, $1.5 \mathrm{~Hz}, 1 \mathrm{H}), 2.10(\mathrm{dd}, J=13.5,9.0 \mathrm{~Hz}, 1 \mathrm{H}), 1.37(\mathrm{~s}, 3 \mathrm{H}), 1.27(\mathrm{~s}, 3 \mathrm{H})$ ppm. ${ }^{13} \mathrm{C}$ NMR $\left(101 \mathrm{MHz}, \mathrm{CDCl}_{3}\right) \delta 172.0,171.9,149.8,148.5$, $131.6,126.9,124.4,116.6,110.6,109.8,58.7,58.3,52.8,52.7,49.6$, 48.5, 44.0, 36.2, 28.1, 21.4, 21.0 ppm. HRMS-ESI calcd for $\mathrm{C}_{21} \mathrm{H}_{28} \mathrm{NO}_{4}^{+}[\mathrm{M}+\mathrm{H}]^{+}: 358.2013$; found: 358.2025.

Dimethyl 3-(2-(3,4-Dihydroquinolin-1(2H)-yl)propan-2-yl)-4methylenecyclopentane-1,1-dicarboxylate (4ag). Obtained using catalyst A (11 mg, $15.0 \mu \mathrm{mol})$, enyne $1 \mathrm{c}(0.29 \mathrm{~mL}, 1.0 \mathrm{M}$ in dry $\left.\mathrm{CH}_{2} \mathrm{Cl}_{2}, 0.29 \mathrm{mmol}\right)$, and 1,2,3,4-tetrahydroquinoline $(0.29 \mathrm{~mL}, 1.0 \mathrm{M}$ in dry $\left.\mathrm{CH}_{2} \mathrm{Cl}_{2}, 0.29 \mathrm{mmol}\right)$ as the nucleophile in $\mathrm{CH}_{2} \mathrm{Cl}_{2}(1.5 \mathrm{~mL})$ according to General Procedure I. Washing with $10 \%$ aqueous solution of $\mathrm{NaOH}$ to remove excess 1,2,3,4-tetrahydroquinoline, flash column chromatography (c-hexane/EtOAc 5:1), and subsequent preparative TLC $\left(\mathrm{CH}_{2} \mathrm{Cl}_{2} / \mathrm{c}\right.$-hexane $\left.1: 2\right)$ yielded $56 \mathrm{mg}(51 \%)$ of the aminated product as a colorless oil. ${ }^{1} \mathrm{H}$ NMR $\left(400 \mathrm{MHz}, \mathrm{CDCl}_{3}\right) \delta 7.06-7.04$ $(\mathrm{m}, 2 \mathrm{H}), 7.01(\mathrm{~d}, J=7.5 \mathrm{~Hz}, 1 \mathrm{H}), 6.74(\mathrm{dt}, J=6.5,2.5 \mathrm{~Hz}, 1 \mathrm{H}), 5.06$ $(\mathrm{s}, 1 \mathrm{H}), 4.98(\mathrm{~s}, 1 \mathrm{H}), 3.75(\mathrm{~s}, 3 \mathrm{H}), 3.72(\mathrm{~s}, 3 \mathrm{H}), 3.62(\mathrm{dt}, J=10.0,2.0$ $\mathrm{Hz}, 1 \mathrm{H}), 3.33-3.28(\mathrm{~m}, 1 \mathrm{H}), 3.23-3.18(\mathrm{~m}, 1 \mathrm{H}), 2.97-2.89(\mathrm{~m}, 2 \mathrm{H})$, $2.65(\mathrm{t}, \mathrm{J}=6.5 \mathrm{~Hz}, 2 \mathrm{H}), 2.55(\mathrm{ddd}, J=13.6,8.0,1.5 \mathrm{~Hz}, 1 \mathrm{H}), 2.06(\mathrm{dd}$, $J=13.5,9.5 \mathrm{~Hz}, 1 \mathrm{H}$ ), 1.89 (quin, $J=6.5 \mathrm{~Hz}, 2 \mathrm{H}), 1.43(\mathrm{~s}, 3 \mathrm{H}), 1.32$ (s, 3H) ppm. ${ }^{13} \mathrm{C}$ NMR (101 MHz, $\left.\mathrm{CDCl}_{3}\right) \delta 172.1,171.9,148.9$, $145.9,131.1,128.4,125.4,119.8,118.3,110.5,60.8,58.4,52.8,52.7$, 48.8, 44.3, 44.1, 36.6, 28.0, 25.3, 24.3, 23.6 ppm. HRMS-ESI calcd for $\mathrm{C}_{22} \mathrm{H}_{30} \mathrm{NO}_{4}^{+}[\mathrm{M}+\mathrm{H}]^{+}: 372.2169$; found: 372.2162 .

4-Bromo-N-((8,8-dimethyl-3-methylene-7,9-dioxaspiro[4,5]decan-2-yl)(phenyl)methyl)aniline (4ah). Obtained using catalyst A (4.6 $\mathrm{mg}, 0.006 \mathrm{mmol})$, enyne $1 \mathrm{~d}(81 \mathrm{mg}, 0.30 \mathrm{mmol})$, and 4 bromoaniline $(57 \mathrm{mg}, 0.33 \mathrm{mmol})$ as the nucleophile in $\mathrm{CH}_{2} \mathrm{Cl}_{2}(0.9$ $\mathrm{mL}$ ) according to General Procedure I (stirred vigorously at rt for 5 h). Purification by column chromatography (pentane/ $\mathrm{Et}_{2} \mathrm{O} 95: 5$ to $9: 1$ ) yielded $125 \mathrm{mg}$ of colorless gum (94\%, contains ca. 3-5\% impurities) that was further crystallized from $0.4 \mathrm{~mL}$ of diethyl ether layered with $1.2 \mathrm{~mL}$ of pentane (sonication initiated the crystallization) to yield $110 \mathrm{mg}$ of colorless crystals (84\%). ${ }^{1} \mathbf{H}$ NMR $\left(500 \mathrm{MHz}, \mathrm{CDCl}_{3}\right) \delta 7.32$ (app. d, $J=4.3 \mathrm{~Hz}, 4 \mathrm{H}$ ), 7.25 (ddd, $J$ $=8.7,4.9,3.9 \mathrm{~Hz}, 1 \mathrm{H}), 7.17-7.12(\mathrm{~m}, 2 \mathrm{H}), 6.40-6.35(\mathrm{~m}, 2 \mathrm{H}), 5.07$ (br s, $1 \mathrm{H}), 4.77(\mathrm{q}, J=2.1 \mathrm{~Hz}, 1 \mathrm{H}), 4.43(\mathrm{~d}, J=5.2 \mathrm{~Hz}, 1 \mathrm{H}), 4.02(\mathrm{br}$ s, $1 \mathrm{H}), 3.72(\mathrm{~d}, J=11.2 \mathrm{~Hz}, 1 \mathrm{H}), 3.66(\mathrm{~d}, J=11.4 \mathrm{~Hz}, 1 \mathrm{H}), 3.55-3.48$ (m, $2 \mathrm{H}), 2.99$ (dddt, $J=10.6,7.9,5.2,2.2 \mathrm{~Hz}, 1 \mathrm{H}), 2.53(\mathrm{dq}, J=16.5$, $1.6 \mathrm{~Hz}, 1 \mathrm{H}), 2.18(\mathrm{dq}, J=16.4,2.4 \mathrm{~Hz}, 1 \mathrm{H}), 1.67(\mathrm{dd}, J=13.6,8.2 \mathrm{~Hz}$, $1 \mathrm{H}), 1.50(\mathrm{dd}, J=13.6,10.3 \mathrm{~Hz}, 1 \mathrm{H}), 1.40(\mathrm{~s}, 3 \mathrm{H}), 1.38(\mathrm{~s}, 3 \mathrm{H}) \mathrm{ppm}$. ${ }^{13} \mathrm{C}$ NMR $\left(126 \mathrm{MHz}, \mathrm{CDCl}_{3}\right) \delta 149.0,146.7,141.7,131.7,128.5$, $127.2,126.8,115.5,109.5,109.5,97.9,69.4,67.5,59.9,48.8,41.7,39.4$, 34.0, 24.7, 22.8 ppm. HRMS-ESI calcd for $\mathrm{C}_{24} \mathrm{H}_{28} \mathrm{BrNO}_{2} \mathrm{Na}^{+}[\mathrm{M}+$ $\mathrm{Na}]^{+}$: 464.1196; found: 464.1197. Mp $110-112{ }^{\circ} \mathrm{C}$.

$\mathrm{N}$-((4,4-Bis(methoxymethyl)-2-methylenecyclopropentyl(phenyl)methyl)-4-bromoaniline (4ai). Obtained using catalyst A (3.9 mg, $0.005 \mathrm{mmol})$, enyne 1f $(64.6 \mathrm{mg}, 0.25 \mathrm{mmol})$, and 4-bromoaniline (47 $\mathrm{mg}, 0.275 \mathrm{mmol}$ ) as the nucleophile in $\mathrm{CH}_{2} \mathrm{Cl}_{2}(1.0 \mathrm{~mL})$ according to General Procedure I (stirred vigorously at r.t. for $5 \mathrm{~h}$ ). Purification by column chromatography (pentane $/ \mathrm{Et}_{2} \mathrm{O} 95: 5$ to $85: 15$ ) yielded $80 \mathrm{mg}$ (74\%) of the aminated product as a colorless oil. ${ }^{1} \mathbf{H}$ NMR $(500 \mathrm{MHz}$, $\left.\mathrm{CDCl}_{3}\right) \delta 7.35-7.28(\mathrm{~m}, 4 \mathrm{H}), 7.25-7.20(\mathrm{~m}, 1 \mathrm{H}), 7.15-7.11(\mathrm{~m}$, $2 \mathrm{H}), 6.37-6.32(\mathrm{~m}, 2 \mathrm{H}), 4.98(\mathrm{q}, J=2.1 \mathrm{~Hz}, 1 \mathrm{H}), 4.75(\mathrm{q}, J=2.1 \mathrm{~Hz}$,
$1 \mathrm{H}), 4.50-4.46(\mathrm{~m}, 2 \mathrm{H}), 3.41(\mathrm{~s}, 3 \mathrm{H}), 3.32(\mathrm{~s}, 2 \mathrm{H}), 3.27(\mathrm{~s}, 3 \mathrm{H}), 3.12$ $(\mathrm{s}, 2 \mathrm{H}), 3.02(\mathrm{t}, J=8.2 \mathrm{~Hz}, 1 \mathrm{H}), 2.40(\mathrm{dq}, J=16.3,2.3 \mathrm{~Hz}, 1 \mathrm{H}), 2.31$ $(\mathrm{d}, J=16.2 \mathrm{~Hz}, 1 \mathrm{H}), 1.75(\mathrm{dd}, J=13.9,9.1 \mathrm{~Hz}, 1 \mathrm{H}), 1.51(\mathrm{dd}, J=$ 13.9, $9.3 \mathrm{~Hz}, 1 \mathrm{H}) \mathrm{ppm} .{ }^{13} \mathrm{C}$ NMR $\left(126 \mathrm{MHz}, \mathrm{CDCl}_{3}\right) \delta 151.2,147.0$, 142.3, 131.6, 128.5, 126.9, 126.7, 115.1, 108.7, 107.7, 77.7, 76.2, 60.4, 59.3, 59.3, 49.7, 45.4, 40.5, $32.2 \mathrm{ppm}$. HRMS-ESI calcd for $\mathrm{C}_{23} \mathrm{H}_{28} \mathrm{BrNO}_{2} \mathrm{Na}^{+}[\mathrm{M}+\mathrm{Na}]^{+}$: 452.1196; found: 452.1197 .

$\mathrm{N}$-((4,4-Bis(((4-methoxybenzyl)oxy)methyl)-2-methylenecyclopentyl)(phenyl)methyl)-4-bromoaniline (4aj). Obtained using catalyst A (2.0 mg, $0.0026 \mathrm{mmol})$, enyne $1 \mathrm{e}(61 \mathrm{mg}, 0.13 \mathrm{mmol})$, and 4 bromoaniline $(57 \mathrm{mg}, 0.143 \mathrm{mmol})$ as the nucleophile in $\mathrm{CH}_{2} \mathrm{Cl}_{2}(0.6$ $\mathrm{mL}$ ) according to General Procedure I (stirred vigorously at $\mathrm{rt}$ for 5 h). Purification by column chromatography (pentane $/ \mathrm{Et}_{2} \mathrm{O} 95: 5$ to $85: 15)$ yielded $61 \mathrm{mg}(73 \%)$ of the aminated product as a colorless oil. ${ }^{1} \mathrm{H}$ NMR $\left(500 \mathrm{MHz}, \mathrm{CDCl}_{3}\right) \delta 7.34-7.21(\mathrm{~m}, 7 \mathrm{H}), 7.18-7.13(\mathrm{~m}$, $2 \mathrm{H}), 7.10-7.06(\mathrm{~m}, 2 \mathrm{H}), 6.92-6.88(\mathrm{~m}, 2 \mathrm{H}), 6.87-6.83(\mathrm{~m}, 2 \mathrm{H})$, $6.20-6.15(\mathrm{~m}, 2 \mathrm{H}), 4.97(\mathrm{q}, J=2.1 \mathrm{~Hz}, 1 \mathrm{H}), 4.74(\mathrm{q}, J=2.1 \mathrm{~Hz}, 1 \mathrm{H})$, $4.49(\mathrm{~s}, 2 \mathrm{H}), 4.47(\mathrm{t}, J 3.0 \mathrm{~Hz}, 1 \mathrm{H}), 4.37(\mathrm{~s}, 2 \mathrm{H}), 4.34(\mathrm{br} \mathrm{s}, 1 \mathrm{H}), 3.83$ (s, $3 \mathrm{H}), 3.82(\mathrm{~s}, 3 \mathrm{H}), 3.46-3.39(\mathrm{~m}, 2 \mathrm{H}), 3.23-3.17(\mathrm{~m}, 2 \mathrm{H}), 2.99$ (dddt, $J=9.3,6.9,4.5,2.2 \mathrm{~Hz}, 1 \mathrm{H}), 2.46(\mathrm{dq}, J=16.4,2.3 \mathrm{~Hz}, 1 \mathrm{H})$, $2.35(\mathrm{~d}, J=16.2 \mathrm{~Hz}, 1 \mathrm{H}), 1.78(\mathrm{dd}, J=14.0,9.2 \mathrm{~Hz}, 1 \mathrm{H}), 1.52(\mathrm{dd}, J=$ 13.9, $9.3 \mathrm{~Hz}, 1 \mathrm{H}) \mathrm{ppm} .{ }^{13} \mathrm{C}$ NMR $\left(126 \mathrm{MHz}, \mathrm{CDCl}_{3}\right) \delta 159.2,159.0$, $151.5,146.9,142.3,131.5,130.6,129.3,128.9,128.4,126.9,126.6$, $115.1,113.8,113.7,108.7,107.5,74.9,73.3,73.1,72.8,60.3,55.3,55.2$, 49.7, 45.5, 40.6, $32.1 \mathrm{ppm}$. HRMS-ESI calcd for $\mathrm{C}_{37} \mathrm{H}_{40} \mathrm{BrNO}_{4} \mathrm{Na}^{+}[\mathrm{M}$ $+\mathrm{Na}]^{+}: 664.2033$; found: 664.2030 .

Dimethyl 9b-Methyl-4-phenyl-1,3,3a,9b-tetrahydro-2H-cyclopenta[c]quinoline-2,2-dicarboxylate (5e). A solution of compound 4e $(40 \mathrm{mg}, 0.08 \mathrm{mmol})$ in degassed DMF $(0.5 \mathrm{~mL})$ was treated with $\mathrm{Pd}(\mathrm{OAc})_{2}(1.8 \mathrm{mg}, 8 \mu \mathrm{mol}), \mathrm{PPh}_{3}(4.2 \mathrm{mg}, 16 \mu \mathrm{mol})$, and $\mathrm{K}_{2} \mathrm{CO}_{3}(22$ $\mathrm{mg}, 0.16 \mathrm{mmol}$ ) under a $\mathrm{N}_{2}$ atmosphere, and the solution was heated at $110{ }^{\circ} \mathrm{C}$ for $7 \mathrm{~h}$. Purification by flash column chromatography (pentane/ $\left.\mathrm{Et}_{2} \mathrm{O} 9: 1\right)$ provided product $5 \mathrm{e}(20 \mathrm{mg}, 67 \%)$ as a colorless oil. ${ }^{1}$ H NMR $\left(500 \mathrm{MHz}, \mathrm{CDCl}_{3}\right) \delta 8.12-8.07(\mathrm{~m}, 2 \mathrm{H}), 7.54-7.45$ $(\mathrm{m}, 4 \mathrm{H}), 7.38(\mathrm{dd}, J=7.5,1.6 \mathrm{~Hz}, 1 \mathrm{H}), 7.28(\mathrm{td}, J=7.5,1.6 \mathrm{~Hz}, 1 \mathrm{H})$, $7.23(\mathrm{td}, J=7.4,1.5 \mathrm{~Hz}, 1 \mathrm{H}), 3.79(\mathrm{~s}, 3 \mathrm{H}), 3.50(\mathrm{~s}, 3 \mathrm{H}), 3.45(\mathrm{~d}, J=$ $14.2 \mathrm{~Hz}, 1 \mathrm{H}), 3.31(\mathrm{dd}, J=12.4,7.8 \mathrm{~Hz}, 1 \mathrm{H}), 2.66(\mathrm{dd}, J=13.8,7.8$ $\mathrm{Hz}, 1 \mathrm{H}), 2.54(\mathrm{~d}, J=14.2 \mathrm{~Hz}, 1 \mathrm{H}), 2.17(\mathrm{dd}, J=13.8,12.4 \mathrm{~Hz}, 1 \mathrm{H})$, $1.20(\mathrm{~s}, 3 \mathrm{H}) \mathrm{ppm} .{ }^{13} \mathrm{C}$ NMR $\left(126 \mathrm{MHz}, \mathrm{CDCl}_{3}\right) \delta 173.4,171.4,165.8$, $142.4,138.7,131.7,130.8,128.8,128.6,127.9,127.7,127.0,125.7$, 58.5, 53.2, 52.9, 46.9, 46.5, 44.5, 38.8, 28.7 ppm. HRMS-ESI calcd for $\mathrm{C}_{23} \mathrm{H}_{24} \mathrm{NO}_{4}^{+}[\mathrm{M}+\mathrm{H}]^{+}$: 378.1700; found: 378.1697.

Dimethyl 8-Bromo-9b-methyl-4-phenyl-1,3,3a,9b-tetrahydro-2Hcyclopenta[c]quinoline-2,2-dicarboxylate (5f). A solution of compound $4 \mathrm{f}(60 \mathrm{mg}, 0.11 \mathrm{mmol})$ in degassed DMF $(0.7 \mathrm{~mL})$ was treated with $\mathrm{Pd}(\mathrm{OAc})_{2}(2.3 \mathrm{mg}, 10 \mu \mathrm{mol}), \mathrm{PPh}_{3}(5.4 \mathrm{mg}, 21 \mu \mathrm{mol})$, and $\mathrm{K}_{2} \mathrm{CO}_{3}(28 \mathrm{mg}, 0.21 \mathrm{mmol})$ under a $\mathrm{N}_{2}$ atmosphere, and the solution was heated at $110{ }^{\circ} \mathrm{C}$ for $4 \mathrm{~h}$. Purification by flash column chromatography (pentane $/ \mathrm{Et}_{2} \mathrm{O}$ 9:1) provided product 5 f $(34 \mathrm{mg}$, $73 \%)$ as a colorless oil. ${ }^{1} \mathbf{H}$ NMR $\left(500 \mathrm{MHz}, \mathrm{CDCl}_{3}\right) \delta 8.15-8.04(\mathrm{~m}$, $2 \mathrm{H}), 7.52-7.45(\mathrm{~m}, 4 \mathrm{H}), 7.42-7.35(\mathrm{~m}, 2 \mathrm{H}), 3.78(\mathrm{~s}, 3 \mathrm{H}), 3.58(\mathrm{~s}$, $3 \mathrm{H}), 3.33(\mathrm{~d}, J=14.3 \mathrm{~Hz}, 1 \mathrm{H}), 3.28(\mathrm{dd}, J=12.2,8.1 \mathrm{~Hz}, 1 \mathrm{H}), 2.63$ $(\mathrm{dd}, J=13.9,8.1 \mathrm{~Hz}, 1 \mathrm{H}), 2.58(\mathrm{~d}, J=14.3 \mathrm{~Hz}, 1 \mathrm{H}), 2.21(\mathrm{dd}, J=$ $13.9,12.2 \mathrm{~Hz}, 1 \mathrm{H}), 1.20(\mathrm{~s}, 3 \mathrm{H}) \mathrm{ppm} .{ }^{13} \mathrm{C}$ NMR $\left(126 \mathrm{MHz} \mathrm{CDCl}_{3}\right) \delta$ $173.1,171.2,166.4,141.6,138.3,133.9,131.1,130.9,130.2,128.8$, 128.8, 127.0, 120.9, 58.5, 53.3, 53.1, 46.8, 46.1, 44.7, 38.9, 28.4 ppm. HRMS-ESI calcd for $\mathrm{C}_{23} \mathrm{H}_{23} \mathrm{BrNO}_{4}^{+}[\mathrm{M}+\mathrm{H}]^{+}$: 456.0805; found: 456.0798 .

Dimethyl 7-Chloro-9b-methyl-4-phenyl-1,3,3a,9b-tetrahydro-2Hcyclopenta[c]quinoline-2,2-dicarboxylate $(5 \mathrm{~g})$. A solution of compound $4 \mathrm{~g}(60 \mathrm{mg}, 0.11 \mathrm{mmol})$ in degassed DMF $(0.75 \mathrm{~mL})$ was treated with $\mathrm{Pd}(\mathrm{OAc})_{2}(2.5 \mathrm{mg}, 11 \mu \mathrm{mol}), \mathrm{PPh}_{3}(5.8 \mathrm{mg}, 22 \mu \mathrm{mol})$, and $\mathrm{K}_{2} \mathrm{CO}_{3}(31 \mathrm{mg}, 0.22 \mathrm{mmol})$ under a $\mathrm{N}_{2}$ atmosphere, and the solution was heated at $110{ }^{\circ} \mathrm{C}$ for $4 \mathrm{~h}$. Purification by flash column chromatography (pentane $/ \mathrm{Et}_{2} \mathrm{O}$ 9:1) provided product $5 \mathrm{~g}(33 \mathrm{mg}$, $72 \%)$ as a colorless oil. ${ }^{1} \mathbf{H}$ NMR $\left(500 \mathrm{MHz}, \mathrm{CDCl}_{3}\right) \delta 8.12-8.06(\mathrm{~m}$, $2 \mathrm{H}), 7.57-7.44(\mathrm{~m}, 4 \mathrm{H}), 7.31(\mathrm{~d}, J=8.2 \mathrm{~Hz}, 1 \mathrm{H}), 7.20(\mathrm{dd}, J=8.2$, $2.3 \mathrm{~Hz}, 1 \mathrm{H}), 3.79(\mathrm{~s}, 3 \mathrm{H}), 3.53(\mathrm{~s}, 3 \mathrm{H}), 3.42(\mathrm{~d}, J=14.3 \mathrm{~Hz}, 1 \mathrm{H}), 3.32$ $(\mathrm{dd}, J=12.4,7.7 \mathrm{~Hz}, 1 \mathrm{H}), 2.68(\mathrm{dd}, J=13.8,7.7 \mathrm{~Hz}, 1 \mathrm{H}), 2.50(\mathrm{~d}, J=$ $14.4 \mathrm{~Hz}, 1 \mathrm{H}), 2.10(\mathrm{dd}, J=13.8,12.4 \mathrm{~Hz}, 1 \mathrm{H}), 1.18(\mathrm{~s}, 3 \mathrm{H}) \mathrm{ppm} .{ }^{13} \mathrm{C}$ 
NMR $\left(126 \mathrm{MHz}, \mathrm{CDCl}_{3}\right) \delta 173.2,171.3,167.3,143.7,138.2,133.0$, $131.2,130.3,128.8,128.4,127.6,127.1,127.0,58.4,53.3,53.0,46.7$, 46.4, 44.4, 38.9, $28.6 \mathrm{ppm}$. HRMS-ESI calcd for $\mathrm{C}_{23} \mathrm{H}_{23} \mathrm{ClNO}_{4}^{+}[\mathrm{M}+$ $\mathrm{H}]^{+}$: 412.1310; found: 412.1320 .

Dimethyl 3-Methylene-4-(phenyl((2-((trimethylsilyl)ethynyl)phenyl)amino)methyl)cyclopentane-1,1-dicarboxylate (6e). A dry $10 \mathrm{~mL}$ MW vial was charged with $\mathrm{PdCl}_{2}\left(\mathrm{PPh}_{3}\right)_{2}(56 \mathrm{mg}, 0.079 \mathrm{mmol})$, $\mathrm{CuI}(38 \mathrm{mg}, 0.20 \mathrm{mmol})$, and 2-iodoaniline $4 \mathrm{e}(500 \mathrm{mg}, 0.99 \mathrm{mmol})$, and the MW vial was sealed and left under argon. Degassed $\mathrm{Et}_{3} \mathrm{~N}$ (7 $\mathrm{mL})$ and degassed DMF $(3 \mathrm{~mL})$ were added followed by trimethylsilylacetylene $(0.68 \mathrm{~mL}, 4.95 \mathrm{mmol}, 5$ equiv $)$ at $25{ }^{\circ} \mathrm{C}$. The resulting mixture was stirred at $25^{\circ} \mathrm{C}$ for $1.5 \mathrm{~h}$ (complete conversion monitored by GC-MS). The dark brown mixture was partitioned between water $(50 \mathrm{~mL})$ and diethyl ether $(20 \mathrm{~mL})$. The aqueous layer was re-extracted with diethyl ether $(2 \times 20 \mathrm{~mL})$. The combined ethereal extracts were dried over sodium sulfate and concentrated. The title product was obtained after purification by chromatography on silica gel eluting with pentane/ $/ \mathrm{Et}_{2} \mathrm{O} 9: 1$ to $4: 1$ as a pale brown crystalline solid (451 mg, 96\%). ${ }^{1} \mathbf{H}$ NMR $\left(500 \mathrm{MHz}, \mathrm{CDCl}_{3}\right) \delta 7.38-$ $7.22(\mathrm{~m}, 6 \mathrm{H}), 6.99(\mathrm{ddd}, J=8.6,7.4,1.6 \mathrm{~Hz}, 1 \mathrm{H}), 6.56(\mathrm{td}, J=7.5,1.0$ $\mathrm{Hz}, 1 \mathrm{H}), 6.30(\mathrm{~d}, J=8.3 \mathrm{~Hz}, 1 \mathrm{H}), 5.14(\mathrm{~d}, J=4.9 \mathrm{~Hz}, 1 \mathrm{H}), 5.08(\mathrm{q}, J$ $=2.6 \mathrm{~Hz}, 1 \mathrm{H}), 4.72(\mathrm{q}, J=2.5 \mathrm{~Hz}, 1 \mathrm{H}), 4.51(\mathrm{t}, J=5.4 \mathrm{~Hz}, 1 \mathrm{H}), 3.70$ $(\mathrm{s}, 3 \mathrm{H}), 3.70(\mathrm{~s}, 3 \mathrm{H}), 3.18-3.10(\mathrm{~m}, 1 \mathrm{H}), 3.04(\mathrm{dq}, J=16.4,1.6 \mathrm{~Hz}$, $1 \mathrm{H}), 2.95(\mathrm{dq}, J=16.5,2.6 \mathrm{~Hz}, 1 \mathrm{H}), 2.43(\mathrm{ddd}, J=13.6,7.8,1.7 \mathrm{~Hz}$, $1 \mathrm{H}), 2.22(\mathrm{dd}, J=13.5,10.4 \mathrm{~Hz}, 1 \mathrm{H}), 0.30(\mathrm{~s}, 9 \mathrm{H}) \mathrm{ppm} .{ }^{13} \mathrm{C}$ NMR $\left(126 \mathrm{MHz}, \mathrm{CDCl}_{3}\right) \delta 171.7,171.4,148.6,146.6,141.5,132.1,129.9$, $128.5,127.2,126.9,116.6,111.1,110.0,108.0,102.0,100.2,59.8,58.0$, 52.8, 52.7, 49.3, 41.8, 35.4, - $0.1 \mathrm{ppm}$. HRMS-ESI calcd for $\mathrm{C}_{28} \mathrm{H}_{34} \mathrm{NO}_{4} \mathrm{Si}^{+}[\mathrm{M}+\mathrm{H}]^{+}$: 476.2252; found: 476.2247. Mp 108-109 ${ }^{\circ} \mathrm{C}$.

Dimethyl 3-(((4-Bromo-2-((trimethylsilyl)ethynyl)phenyl)amino)(phenyl)methyl)-4-methylenecyclopentane-1,1-dicarboxylate (6f). Prepared according to the procedure for $6 \mathrm{e}$, with $4 \mathrm{ff}(440 \mathrm{mg}, 0.753$ $\mathrm{mmol}), \mathrm{PdCl}_{2}\left(\mathrm{PPh}_{3}\right)_{2}$ (42 mg, $\left.0.06 \mathrm{mmol}\right)$, and $\mathrm{CuI}(29 \mathrm{mg}, 0.15$ $\mathrm{mmol})$ in degassed $\mathrm{DFM} / \mathrm{Et}_{3} \mathrm{~N}(1: 2,6.75 \mathrm{~mL})$ to which was added TMS-acetylene ( $208 \mu \mathrm{L}, 1.51 \mathrm{mmol}, 2$ equiv), followed by stirring at $25{ }^{\circ} \mathrm{C}$ for $1.5 \mathrm{~h}$. Obtained as a pale yellow oil after purification by column chromatography on silica gel eluting with pentane $/ \mathrm{Et}_{2} \mathrm{O} 9: 1$ to 4:1 (400 mg, 96\%). ${ }^{1} \mathrm{H}$ NMR $\left(500 \mathrm{MHz}, \mathrm{CDCl}_{3}\right) \delta 7.38-7.31(\mathrm{~m}$, $4 \mathrm{H}), 7.30-7.24(\mathrm{~m}, 1 \mathrm{H}), 7.19(\mathrm{~d}, J=8.2 \mathrm{~Hz}, 1 \mathrm{H}), 6.53(\mathrm{dd}, J=8.2$, $2.0 \mathrm{~Hz}, 1 \mathrm{H}), 6.29(\mathrm{~d}, J=1.9 \mathrm{~Hz}, 1 \mathrm{H}), 5.19(\mathrm{~d}, J=5.3 \mathrm{~Hz}, 1 \mathrm{H}), 5.07$ $(\mathrm{dq}, J=2.5,1.5 \mathrm{~Hz}, 1 \mathrm{H}), 4.71-4.68(\mathrm{~m}, 1 \mathrm{H}), 4.48(\mathrm{t}, J=5.6 \mathrm{~Hz}, 1 \mathrm{H})$, $3.70(\mathrm{~s}, 3 \mathrm{H}), 3.69(\mathrm{~s}, 3 \mathrm{H}), 3.19-3.09(\mathrm{~m}, 1 \mathrm{H}), 3.02(\mathrm{dq}, J=16.4,1.6$ $\mathrm{Hz}, 1 \mathrm{H}), 2.91(\mathrm{dq}, J=16.5,2.6 \mathrm{~Hz}, 1 \mathrm{H}), 2.43(\mathrm{ddd}, J=13.5,7.8,1.7$ $\mathrm{Hz}, 1 \mathrm{H}), 2.16$ (dd, $J=13.5,10.4 \mathrm{~Hz}, 1 \mathrm{H}), 0.31(\mathrm{~s}, 9 \mathrm{H}) \mathrm{ppm} .{ }^{13} \mathrm{C}$ NMR $\left(126 \mathrm{MHz}, \mathrm{CDCl}_{3}\right) \delta 171.7,171.4,149.4,146.5,140.7,135.7$, $132.9,128.6,127.5,126.8,116.7,111.1,110.2,106.6,101.2,100.9$, 59.7, 58.0, 52.8, 52,8, 49.1, 41.8, 35.8, 0.0 ppm. HRMS-ESI calcd for $\mathrm{C}_{28} \mathrm{H}_{32} \mathrm{BrNO}_{4} \mathrm{SiNa}^{+}[\mathrm{M}+\mathrm{Na}]^{+}$: 576.1176; found: 576.1196 .

Dimethyl 3-(((4-Chloro-2-((trimethylsilyl)ethynyl)phenyl)amino)(phenyl)methyl)-4-methylenecyclopentane-1,1-dicarboxylate (6g). Prepared according to the procedure for $6 \mathbf{e}$, with $4 \mathrm{~g}(550 \mathrm{mg}, 1.02$ $\mathrm{mmol}), \mathrm{PdCl}_{2}\left(\mathrm{PPh}_{3}\right)_{2}(57.2 \mathrm{mg}, 0.08 \mathrm{mmol}), \mathrm{CuI}(39 \mathrm{mg}, 0.20 \mathrm{mmol})$ in degassed $\mathrm{DFM} / \mathrm{Et}_{3} \mathrm{~N}(1: 2,7.5 \mathrm{~mL})$ to which was added TMSacetylene ( $282 \mu \mathrm{L}, 2.04 \mathrm{mmol}, 2$ equiv), followed by stirring at $25{ }^{\circ} \mathrm{C}$ for $1.5 \mathrm{~h}$. Obtained as a yellow oil after purification by column chromatography on silica gel eluting with pentane $/ \mathrm{Et}_{2} \mathrm{O} 9: 1$ to $4: 1$ (502 mg, 97\%). ${ }^{1} \mathrm{H}$ NMR (500 MHz, $\left.\mathrm{CDCl}_{3}\right) \delta 7.39(\mathrm{~d}, J=2.4 \mathrm{~Hz}$, $1 \mathrm{H}), 7.36-7.30(\mathrm{~m}, 4 \mathrm{H}), 7.28-7.23(\mathrm{~m}, 1 \mathrm{H}), 7.05(\mathrm{dd}, J=8.8,2.3$ $\mathrm{Hz}, 1 \mathrm{H}), 6.16(\mathrm{~d}, J=8.8 \mathrm{~Hz}, 1 \mathrm{H}), 5.11(\mathrm{~d}, J=4.8 \mathrm{~Hz}, 1 \mathrm{H}), 5.08(\mathrm{q}, J$ $=2.5 \mathrm{~Hz}, 1 \mathrm{H}), 4.71(\mathrm{q}, J=2.5 \mathrm{~Hz}, 1 \mathrm{H}), 4.47(\mathrm{t}, J=5.3 \mathrm{~Hz}, 1 \mathrm{H}), 3.70$ (s, 3H), $3.69(\mathrm{~s}, 3 \mathrm{H}), 3.13$ (dddd, $J=10.4,8.0,5.6,2.3 \mathrm{~Hz}, 1 \mathrm{H}), 3.03$ $(\mathrm{dq}, J=16.5,1.6 \mathrm{~Hz}, 1 \mathrm{H}), 2.92(\mathrm{dq}, J=16.5,2.6 \mathrm{~Hz}, 1 \mathrm{H}), 2.40(\mathrm{ddd}, J$ $=13.6,7.8,1.7 \mathrm{~Hz}, 1 \mathrm{H}), 2.18(\mathrm{dd}, J=13.5,10.5 \mathrm{~Hz}, 1 \mathrm{H}), 0.30(\mathrm{~s}, 9 \mathrm{H})$ ppm. ${ }^{13} \mathrm{C}$ NMR $\left(126 \mathrm{MHz}, \mathrm{CDCl}_{3}\right) \delta 171.7,171.4,147.5,146.5$, $140.9,134.2,132.5,128.6,127.4,126.8,112.7,110.1,110.0,107.8$, $101.7,100.3,59.8,58.0,52.8,52,8,49.2,41.8,35.7,0.0$ ppm. HRMSESI calcd for $\mathrm{C}_{28} \mathrm{H}_{32} \mathrm{ClNO}_{4} \mathrm{SiNa}^{+}[\mathrm{M}+\mathrm{Na}]^{+}$: 532.1681; found: 532.1693.
Dimethyl 3-(((2-Ethynylphenyl)amino)(phenyl)methyl)-4methylenecyclopentane-1,1-dicarboxylate (7e). Substrate 6e (150 $\mathrm{mg}, 0.32 \mathrm{mmol})$ was suspended in methanol $(1 \mathrm{~mL})$ at $25^{\circ} \mathrm{C}$, and $\mathrm{K}_{2} \mathrm{CO}_{3}(131 \mathrm{mg}, 0.95 \mathrm{mmol})$ was added. The mixture was stirred vigorously at $25{ }^{\circ} \mathrm{C}$ for $3 \mathrm{~h}$ (disappearance of starting material monitored by GC-MS). The volatiles were then removed under a stream of nitrogen, and the residue was purified by column chromatography on silica gel eluting with pentane $/ \mathrm{Et}_{2} \mathrm{O} 4: 1$ to afford $120 \mathrm{mg}$ of colorless oil (94\%). Note: alternatively the deprotection of the TMS group can be carried out on the crude mixture after Sonogashira coupling to afford the title alkyne in $81 \%$ yield over 2 steps $(1 \mathrm{~g}$ scale of iodoaniline substrate $[1.98 \mathrm{mmol}], 650 \mathrm{mg}$ [1.61 $\mathrm{mmol}]$ of alkyne obtained). ${ }^{1} \mathrm{H}$ NMR $\left(500 \mathrm{MHz}, \mathrm{CDCl}_{3}\right) \delta 7.38-7.29$ $(\mathrm{m}, 5 \mathrm{H}), 7.27-7.22(\mathrm{~m}, 1 \mathrm{H}), 7.03(\mathrm{ddd}, J=8.6,7.4,1.6 \mathrm{~Hz}, 1 \mathrm{H}), 6.58$ $(\mathrm{td}, J=7.5,1.0 \mathrm{~Hz}, 1 \mathrm{H}), 6.31(\mathrm{~d}, J=8.4 \mathrm{~Hz}, 1 \mathrm{H}), 5.14(\mathrm{~d}, J=5.1 \mathrm{~Hz}$, $1 \mathrm{H}), 5.07(\mathrm{q}, J=2.1 \mathrm{~Hz}, 1 \mathrm{H}), 4.73(\mathrm{q}, J=2.1 \mathrm{~Hz}, 1 \mathrm{H}), 4.55(\mathrm{t}, J=5.4$ $\mathrm{Hz}, 1 \mathrm{H}), 3.72(\mathrm{~s}, 3 \mathrm{H}), 3.70(\mathrm{~s}, 3 \mathrm{H}), 3.52(\mathrm{~s}, 1 \mathrm{H}), 3.22-3.13(\mathrm{~m}, 1 \mathrm{H})$, $3.08-2.96(\mathrm{~m}, 2 \mathrm{H}), 2.45$ (dd, $J=13.7,8.2 \mathrm{~Hz}, 1 \mathrm{H}), 2.26(\mathrm{dd}, J=13.7$, $9.9 \mathrm{~Hz}, 1 \mathrm{H})$ ppm. ${ }^{13} \mathrm{C}$ NMR $\left(126 \mathrm{MHz}, \mathrm{CDCl}_{3}\right) \delta 171.8,171.7,148.8$, $147.0,141.4,132.3,130.1,128.5,127.2,126.8,116.5,111.0,110.0$, $106.8,83.2,80.7,60.0,58.2,52.8,49.3,42.1,35.4$ ppm. HRMS-ESI calcd for $\mathrm{C}_{25} \mathrm{H}_{25} \mathrm{NO}_{4} \mathrm{Na}^{+}[\mathrm{M}+\mathrm{Na}]^{+}$: 426.1676; found: 426.1681 .

Dimethyl 3-((4-Bromo-2-ethynylphenyl)amino)(phenyl)methyl)4-methylenecyclopentane-1,1-dicarboxylate (7f). Prepared according to the procedure for $7 \mathrm{e}$, with $6 \mathrm{f}(115 \mathrm{mg}, 0.21 \mathrm{mmol})$ and $\mathrm{K}_{2} \mathrm{CO}_{3}$ $(86 \mathrm{mg}, 0.62 \mathrm{mmol})$ in methanol $(0.7 \mathrm{~mL})$, followed by stirring at 25 ${ }^{\circ} \mathrm{C}$ for $1.5 \mathrm{~h}$. Obtained as a colorless oil after purification by column chromatography on silica gel eluting with pentane/ $/ \mathrm{Et}_{2} \mathrm{O} 9: 1$ to $4: 1$ (72 $\mathrm{mg}, 72 \%) .{ }^{1} \mathbf{H}$ NMR $\left(500 \mathrm{MHz}, \mathrm{CDCl}_{3}\right) \delta 7.41(\mathrm{~d}, J=2.4 \mathrm{~Hz}, 1 \mathrm{H})$, 7.35-7.29 (m, 4H), 7.27-7.23 (m, $1 \mathrm{H}), 7.09(\mathrm{dd}, J=8.8,2.4 \mathrm{~Hz}$, $1 \mathrm{H}), 6.17(\mathrm{~d}, J=8.9 \mathrm{~Hz}, 1 \mathrm{H}), 5.13(\mathrm{~d}, J=5.1 \mathrm{~Hz}, 1 \mathrm{H}), 5.07(\mathrm{q}, J=2.1$ $\mathrm{Hz}, 1 \mathrm{H}), 4.71(\mathrm{q}, J=2.1 \mathrm{~Hz}, 1 \mathrm{H}), 4.49(\mathrm{t}, J=5.4 \mathrm{~Hz}, 1 \mathrm{H}), 3.72(\mathrm{~s}$, $3 \mathrm{H}), 3.70(\mathrm{~s}, 3 \mathrm{H}), 3.54(\mathrm{~s}, 1 \mathrm{H}), 3.19-3.12(\mathrm{~m}, 1 \mathrm{H}), 3.05-2.95(\mathrm{~m}$, $2 \mathrm{H}), 2.42(\mathrm{dd}, J=13.7,8.2 \mathrm{~Hz}, 1 \mathrm{H}), 2.21(\mathrm{dd}, J=13.8,9.8 \mathrm{~Hz}, 1 \mathrm{H})$ ppm. ${ }^{13} \mathrm{C}$ NMR (126 MHz, $\left.\mathrm{CDCl}_{3}\right) \delta 171.7,171.7,147.8,146.9$, $140.9,134.4,132.9,128.6,127.4,126.7,112.6,110.0,108.7,107.7$, $84.3,79.2,60.0,58.1,52.9,52.8,49.2,42.2,35.4$ ppm. HRMS-ESI calcd for $\mathrm{C}_{25} \mathrm{H}_{24} \mathrm{BrNO}_{4} \mathrm{Na}^{+}[\mathrm{M}+\mathrm{Na}]^{+}$: 504.0781; found: 504.0778.

Dimethyl 3-(((4-Chloro-2-ethynylphenyl)amino)(phenyl)methyl)4-methylenecyclopentane-1,1-dicarboxylate (7g). Prepared according to the procedure for $7 \mathrm{e}$, with $6 \mathrm{~g}(135 \mathrm{mg}, 0.27 \mathrm{mmol})$ and $\mathrm{K}_{2} \mathrm{CO}_{3}$ $(110 \mathrm{mg}, 0.79 \mathrm{mmol})$ in methanol $(1 \mathrm{~mL})$, followed by stirring at 25 ${ }^{\circ} \mathrm{C}$ for $2.5 \mathrm{~h}$. Obtained as a pale yellow oil after purification by column chromatography on silica gel eluting with pentane $/ \mathrm{Et}_{2} \mathrm{O}$ 9:1 to 4:1 (78 $\mathrm{mg}, 67 \%) .{ }^{1} \mathrm{H}$ NMR $\left(500 \mathrm{MHz}, \mathrm{CDCl}_{3}\right) \delta 7.36-7.31$ (m, 4H), 7.29$7.24(\mathrm{~m}, 1 \mathrm{H}), 7.22(\mathrm{~d}, J=8.1 \mathrm{~Hz}, 1 \mathrm{H}), 6.55(\mathrm{dd}, J=8.2,2.0 \mathrm{~Hz}, 1 \mathrm{H})$, $6.29(\mathrm{~d}, J=1.9 \mathrm{~Hz}, 1 \mathrm{H}), 5.21(\mathrm{~d}, J=5.5 \mathrm{~Hz}, 1 \mathrm{H}), 5.07(\mathrm{q}, J=2.1 \mathrm{~Hz}$, $1 \mathrm{H}), 4.71(\mathrm{q}, J=2.1 \mathrm{~Hz}, 1 \mathrm{H}), 4.51(\mathrm{t}, J=5.6 \mathrm{~Hz}, 1 \mathrm{H}), 3.72(\mathrm{~s}, 3 \mathrm{H})$, $3.70(\mathrm{~s}, 3 \mathrm{H}), 3.54(\mathrm{~s}, 1 \mathrm{H}), 3.20-3.12(\mathrm{~m}, 1 \mathrm{H}), 3.04-2.94(\mathrm{~m}, 2 \mathrm{H})$, $2.44(\mathrm{dd}, J=13.7,8.3 \mathrm{~Hz}, 1 \mathrm{H}), 2.20(\mathrm{dd}, J=13.8,9.8 \mathrm{~Hz}, 1 \mathrm{H}) \mathrm{ppm}$. ${ }^{13} \mathrm{C}$ NMR $\left(126 \mathrm{MHz}, \mathrm{CDCl}_{3}\right) \delta 171.7,171.7,149.6,146.8,140.6$, 136.0, 133.1, 128.7, 127.5, 126.7, 116.7, 111.0, 110.1, 105.3, 84.0, 79.8, $59.9,58.2,52.8,49.2,42.2, \quad 35.4$ ppm. HRMS-ESI calcd for $\mathrm{C}_{25} \mathrm{H}_{24} \mathrm{ClNO}_{4} \mathrm{Na}^{+}[\mathrm{M}+\mathrm{Na}]^{+}$: 460.1286 ; found: 460.1286 .

Dimethyl 3-((1H-Indol-1-yl)(phenyl)methyl)-4-methylenecyclopentane-1,1-dicarboxylate (8e). In a dry vial, $7 \mathrm{e}(100 \mathrm{mg}, 0.25$ $\mathrm{mmol})$ was dissolved in dry $\mathrm{CH}_{2} \mathrm{Cl}_{2}(2.5 \mathrm{~mL})$ and $\operatorname{IPrAuNTf}_{2}\left(\mathbf{D}_{2}\right)$ $(10.7 \mathrm{mg}, 12 \mu \mathrm{mol})$ was added in one portion. The mixture was stirred at $25{ }^{\circ} \mathrm{C}$ for $8 \mathrm{~h}$ (monitored by GC-MS). The solvent was removed under a stream of nitrogen, and the crude mixture was loaded on silica gel and purified by column chromatography on silica gel eluting with pentane/ $\mathrm{Et}_{2} \mathrm{O} 9: 1$ to $4: 1$ to afford the title product as a colorless solid $(78 \mathrm{mg}, 78 \%) .{ }^{1} \mathrm{H}$ NMR $\left(500 \mathrm{MHz}, \mathrm{CDCl}_{3}\right) \delta 7.62(\mathrm{~d}, J=7.9 \mathrm{~Hz}$, $1 \mathrm{H}), 7.47-7.42(\mathrm{~m}, 2 \mathrm{H}), 7.40-7.34(\mathrm{~m}, 2 \mathrm{H}), 7.32-7.18(\mathrm{~m}, 4 \mathrm{H})$, 7.09 (ddd, $J=7.9,7.0,1.0 \mathrm{~Hz}, 1 \mathrm{H}), 6.60(\mathrm{~d}, J=3.2 \mathrm{~Hz}, 1 \mathrm{H}), 5.29$ (d, $J$ $=11.0 \mathrm{~Hz}, 1 \mathrm{H}), 4.90(\mathrm{q}, J=2.0 \mathrm{~Hz}, 1 \mathrm{H}), 4.18-4.14(\mathrm{~m}, 1 \mathrm{H}), 3.87-$ $3.78(\mathrm{~m}, 1 \mathrm{H}), 3.75(\mathrm{~s}, 3 \mathrm{H}), 3.63(\mathrm{~s}, 3 \mathrm{H}), 3.23(\mathrm{dq}, J=16.3,2.4 \mathrm{~Hz}$, $1 \mathrm{H}), 2.97(\mathrm{dq}, J=16.3,1.5 \mathrm{~Hz}, 1 \mathrm{H}), 2.66(\mathrm{ddd}, J=13.9,7.9,1.6 \mathrm{~Hz}$, $1 \mathrm{H}), 1.88(\mathrm{dd}, J=13.8,8.2 \mathrm{~Hz}, 1 \mathrm{H}) \mathrm{ppm} .{ }^{13} \mathrm{C}$ NMR $(126 \mathrm{MHz}$, $\left.\mathrm{CDCl}_{3}\right) \delta 171.9,171.7,146.7,139.8,136.4,128.4,128.3,127.8,127.6$, 
124.6, 121.7, 120.9, 119.6, 111.6, 109.7, 102.5, 63.4, 58.1, 52.9, 52.8, 46.3, 41.7, 38.4 ppm. HRMS-ESI calcd for $\mathrm{C}_{25} \mathrm{H}_{26} \mathrm{NO}_{4}{ }^{+}[\mathrm{M}+\mathrm{H}]^{+}$: 404.1856; found: 404.1853 . Mp 52-54 ${ }^{\circ} \mathrm{C}$.

Dimethyl 3-((5-Bromo-1H-indol-1-yl)(phenyl)methyl)-4methylenecyclopentane-1,1-dicarboxylate (8f). Prepared according to the procedure for $8 \mathbf{e}$, with $\mathbf{7 f}(61 \mathrm{mg}, 0.13 \mathrm{mmol})$ and $\mathrm{IPrAuNTf}_{2}$ $\left(D_{2}\right)(5.5 \mathrm{mg}, 6.3 \mu \mathrm{mol})$ in $\mathrm{CH}_{2} \mathrm{Cl}_{2}(1.2 \mathrm{~mL})$, followed by stirring at $25{ }^{\circ} \mathrm{C}$ for $8 \mathrm{~h}$. Obtained as a colorless foamy solid after purification by column chromatography on silica gel eluting with pentane $/ \mathrm{Et}_{2} \mathrm{O} 9: 1$ to $4: 1(47 \mathrm{mg}, 77 \%) .{ }^{1} \mathrm{H}$ NMR $\left(500 \mathrm{MHz}, \mathrm{CDCl}_{3}\right) \delta 7.71(\mathrm{~d}, J=1.6 \mathrm{~Hz}$, $1 \mathrm{H}), 7.40(\mathrm{~d}, J=3.3 \mathrm{~Hz}, 1 \mathrm{H}), 7.34-7.21(\mathrm{~m}, 7 \mathrm{H}), 6.51(\mathrm{~d}, J=3.5 \mathrm{~Hz}$, $1 \mathrm{H}), 5.22(\mathrm{~d}, J=10.9 \mathrm{~Hz}, 1 \mathrm{H}), 4.89(\mathrm{q}, J=1.9 \mathrm{~Hz}, 1 \mathrm{H}), 4.16(\mathrm{q}, J=$ $1.7 \mathrm{~Hz}, 1 \mathrm{H}), 3.81-3.74(\mathrm{~m}, 1 \mathrm{H}), 3.73(\mathrm{~s}, 3 \mathrm{H}), 3.63(\mathrm{~s}, 3 \mathrm{H}), 3.19(\mathrm{dq}$, $J=16.3,2.3 \mathrm{~Hz}, 1 \mathrm{H}), 2.96(\mathrm{dq}, J=16.4,1.5 \mathrm{~Hz}, 1 \mathrm{H}), 2.59(\mathrm{ddd}, J=$ 13.8, 7.9, $1.5 \mathrm{~Hz}, 1 \mathrm{H}), 1.84$ (dd, $J=13.8,8.2 \mathrm{~Hz}, 1 \mathrm{H}) \mathrm{ppm} .{ }^{13} \mathrm{C}$ NMR $\left(126 \mathrm{MHz}, \mathrm{CDCl}_{3}\right) \delta 171.8,171.7,146.6,139.4,135.0,130.0,128.6$, $128.1,127.5,125.9,124.5,123.4,112.9,111.7,111.2,102.1,63.7,58.0$, 52.9, 52.9, 46.3, 41.6, 38.3 ppm. HRMS-ESI calcd for $\mathrm{C}_{25} \mathrm{H}_{25} \mathrm{BrNO}_{4}^{+}$ $[\mathrm{M}+\mathrm{H}]^{+}$: 482.0961 ; found: $482.0959 . \mathrm{Mp} 55-56{ }^{\circ} \mathrm{C}$.

Dimethyl 3-((5-Chloro-1H-indol-1-yl)(phenyl)methyl)-4methylenecyclopentane-1,1-dicarboxylate (8g). Prepared according to the procedure for $8 \mathrm{e}$, with $7 \mathrm{~g}(62 \mathrm{mg}, 0.14 \mathrm{mmol})$ and IPrAuNTf $\left(D_{2}\right)(6.1 \mathrm{mg}, 7.1 \mu \mathrm{mol})$ in $\mathrm{CH}_{2} \mathrm{Cl}_{2}(1.3 \mathrm{~mL})$, followed by stirring at $25^{\circ} \mathrm{C}$ for $8 \mathrm{~h}$. Obtained as a colorless foamy solid after purification by column chromatography on silica gel eluting with pentane/ $\mathrm{Et}_{2} \mathrm{O} 9: 1$ to 4:1 (42 mg, 68\%). ${ }^{1} \mathbf{H}$ NMR (500 MHz, $\left.\mathrm{CDCl}_{3}\right) \delta 7.49(\mathrm{~d}, J=8.4 \mathrm{~Hz}$, $1 \mathrm{H}), 7.41-7.39(\mathrm{~m}, 2 \mathrm{H}), 7.36-7.33(\mathrm{~m}, 2 \mathrm{H}), 7.32-7.28(\mathrm{~m}, 2 \mathrm{H})$, $7.27-7.22(\mathrm{~m}, 1 \mathrm{H}), 7.04(\mathrm{dd}, J=8.4,1.8 \mathrm{~Hz}, 1 \mathrm{H}), 6.55(\mathrm{dd}, J=3.3$, $1.8 \mathrm{~Hz}, 1 \mathrm{H}), 5.18(\mathrm{~d}, J=11.0 \mathrm{~Hz}, 1 \mathrm{H}), 4.89(\mathrm{q}, J=2.0 \mathrm{~Hz}, 1 \mathrm{H}), 4.14$ (dd, $J=2.7,1.5 \mathrm{~Hz}, 1 \mathrm{H}), 3.81-3.75(\mathrm{~m}, 1 \mathrm{H}), 3.74(\mathrm{~s}, 3 \mathrm{H}), 3.64(\mathrm{~s}$, $3 \mathrm{H}), 3.20(\mathrm{dq}, J=16.4,2.4 \mathrm{~Hz}, 1 \mathrm{H}), 2.96(\mathrm{dq}, J=16.3,1.5 \mathrm{~Hz}, 1 \mathrm{H})$, $2.61(\mathrm{ddd}, J=13.8,7.9,1.6 \mathrm{~Hz}, 1 \mathrm{H}), 1.83(\mathrm{dd}, J=13.8,8.2 \mathrm{~Hz}, 1 \mathrm{H})$ ppm. ${ }^{13} \mathrm{C}$ NMR (126 MHz, $\left.\mathrm{CDCl}_{3}\right) \delta 171.8,171.6,146.5,139.4$, 136.8 , 128.6, 128.1, 127.8, 127.6, 126.8, 125.3, 121.7, 120.3, 111.7, 109.7, 102.8, 63.6, 58.1, 52.9, 52.9, 46.3, 41.6, 38.3 ppm. HRMS-ESI calcd for $\mathrm{C}_{25} \mathrm{H}_{24} \mathrm{ClNO}_{4} \mathrm{Na}^{+}[\mathrm{M}+\mathrm{Na}]^{+}:$460.1286; found: 460.1281 . Mp $49-50{ }^{\circ} \mathrm{C}$.

\section{ASSOCIATED CONTENT}

\section{S Supporting Information}

. The Supporting Information is available free of charge on the ACS Publications website at DOI: 10.1021/acs.joc.5b02607.

Crystallographic data files for $\mathbf{4 i}, \mathbf{4 d}$, and $\mathbf{4 x}$ (ZIP)

Spectral data for all new compounds and X-ray crystallography data for compounds $\mathbf{4 d}, \mathbf{4 i}$, and $\mathbf{4 x}(\mathrm{PDF})$

\section{AUTHOR INFORMATION}

\section{Corresponding Author}

*E-mail: aechavarren@iciq.es.

\section{Notes}

The authors declare no competing financial interest.

\section{ACKNOWLEDGMENTS}

We thank MINECO (Severo Ochoa Excellence Accreditation 2014-2018 (SEV-2013-0319), Project CTQ2013-42106-P), the European Research Council (Advanced Grant No. 321066), the AGAUR (2014 SGR 818 and Beatriu de Pinós Postdoctoral Fellowship to J.C.), and the ICIQ Foundation. M.E.M. and F.C. acknowledge the receipt of COFUND (Marie Curie program) and Swiss National Science Foundation postdoctoral fellowships, respectively. We also thank the ICIQ X-ray diffraction unit for the structures of $\mathbf{4 d}, \mathbf{4 i}$, and $\mathbf{4 x}$.

\section{REFERENCES}

(1) (a) Jiménez-Núñez, E.; Echavarren, A. M. Chem. Rev. 2008, 108, 3326-3350. (b) Fürstner, A. Chem. Soc. Rev. 2009, 38, 3208-3221. (c) Shapiro, N. D.; Toste, F. D. Synlett 2010, 2010, 675-691. (d) Obradors, C.; Echavarren, A. M. Acc. Chem. Res. 2014, 47, 902912. (e) Fensterbank, L.; Malacria, M. Acc. Chem. Res. 2014, 47, 953965. (f) Dorel, R.; Echavarren, A. M. Chem. Rev. 2015, 115, 90289072. (g) Dorel, R.; Echavarren, A. M. J. Org. Chem. 2015, 80, 73217332.

(2) For the addition of $C$-based nucleophiles, see: (a) Toullec, P. Y.; Genin, E.; Leseurre, L.; Genêt, J.-P.; Michelet, V. Angew. Chem., Int. Ed. 2006, 45, 7427-7430. (b) Amijs, C. H. M.; López-Carrillo, V.; Raducan, M.; Pérez-Galán, P.; Ferrer, C.; Echavarren, A. M. J. Org. Chem. 2008, 73, 7721-7730.

(3) (a) Nieto-Oberhuber, C.; Muñoz, M. P.; Buñuel, E.; Nevado, C.; Cárdenas, D. J.; Echavarren, A. M. Angew. Chem., Int. Ed. 2004, 43, 2402-2406. (b) Nieto-Oberhuber, C.; Muñoz, M. P.; López, S.; Jiménez-Núñez, E.; Nevado, C.; Herrero-Gómez, E.; Raducan, M.; Echavarren, A. M. Chem. - Eur. J. 2006, 12, 1677-1693; Corrigendum. Chem. - Eur. J. 2008, 14, 5096.

(4) (a) Horino, Y.; Luzung, M. R.; Toste, F. D. J. Am. Chem. Soc. 2006, 128, 11364-11365. (b) Buzas, A. K.; Istrate, F. M.; Gagosz, F. Angew. Chem., Int. Ed. 2007, 46, 1141-1144. (c) Ricard, L.; Gagosz, F. Organometallics 2007, 26, 4704-4707. (d) Genin, E.; Leseurre, L.; Toullec, P. Y.; Genêt, J. P.; Michelet, V. Synlett 2007, 2007, 17801784. (e) Fürstner, A.; Morency, L. Angew. Chem., Int. Ed. 2008, 47, 5030-5033. (f) Chao, C.-M.; Toullec, P. Y.; Michelet, V. Tetrahedron Lett. 2009, 50, 3719-3722.

(5) (a) Méndez, M.; Muñoz, M. P.; Nevado, C.; Cárdenas, D. J.; Echavarren, A. M. J. Am. Chem. Soc. 2001, 123, 10511-10520. (b) Michelet, V.; Charruault, L.; Gladiali, S.; Genêt, J. P. Pure Appl. Chem. 2006, 78, 397-407 and references therein. (c) Nevado, C.; Charruault, L.; Michelet, V.; Nieto-Oberhuber, C.; Muñoz, M. P.; Méndez, M.; Rager, M.-N.; Genêt, J. P.; Echavarren, A. M. Eur. J. Org. Chem. 2003, 2003, 706-713.

(6) (a) Komeyama, K.; Miyagi, M.; Takaki, K. Heteroat. Chem. 2008, 19, 644-648. (b) Komeyama, K.; Saigo, N.; Miyagi, M.; Takaki, K. Angew. Chem., Int. Ed. 2009, 48, 9875-9878. (c) Komeyama, K.; Takahashi, K.; Takaki, K. Chem. Lett. 2008, 37, 602-603.

(7) Nieto-Oberhuber, C.; López, S.; Muñoz, M. P.; Cárdenas, D. J.; Buñuel, E.; Nevado, C.; Echavarren, A. M. Angew. Chem., Int. Ed. 2005, $44,6146-6148$

(8) (a) Hintermann, L.; Labonne, A. Synthesis 2007, 2007, 11211150. (b) Ghosh, N.; Nayak, S.; Sahoo, A. K. J. Org. Chem. 2011, 76, 500-511.

(9) (a) Muñoz, M. P.; Adrio, J.; Carretero, J. C.; Echavarren, A. M. Organometallics 2005, 24, 1293-1300. (b) Chao, C.-M.; Genin, E.; Toullec, P. Y.; Genêt, J.-P.; Michelet, V. J. Organomet. Chem. 2009, 694, 538-545. (c) Matsumoto, Y.; Selim, K. B.; Nakanishi, H.; Yamada, K.; Yamamoto, Y.; Tomioka, K. Tetrahedron Lett. 2010, 51, 404-406. (d) Wang, W.; Yang, J.; Wang, F.; Shi, M. Organometallics 2011, 30, 3859-3869. (e) Banerjee, D.; Buzas, A. K.; Besnard, C.; Kündig, E. P. Organometallics 2012, 31, 8348-8354.

(10) Zhang, L.; Kozmin, S. A. J. Am. Chem. Soc. 2005, 127, 69626963.

(11) Pradal, A.; Chen, Q.; Bel, P. D.; Toullec, P. Y.; Michelet, V. Synlett 2012, 2012, 74-79.

(12) Cabello, N.; Rodríguez, C.; Echavarren, A. M. Synlett 2007, 2007, 1753-1758.

(13) Leseurre, L.; Toullec, P. Y.; Genêt, J.-P.; Michelet, V. Org. Lett. 2007, 9, 4049-4052.

(14) Ranieri, B.; Escofet, I.; Echavarren, A. M. Org. Biomol. Chem. 2015, 13, 7103-7118.

(15) See Supporting Information for additional results.

(16) Young, P. C.; Green, S. L. J.; Rosair, G. M.; Lee, A.-L. Dalton Trans. 2013, 42, 9645-9653.

(17) CCDC 1436564 (4d), CCDC 1436562 (4i), and CCDC $1436563(4 x)$ contain the supplementary crystallographic data. These data can be obtained free of charge from The Cambridge 
Crystallographic Data Centre via www.ccdc.cam.ac.uk/data_request/ cif.

(18) Vaghi, L.; Benincori, T.; Cirilli, R.; Alberico, E.; Mussini, P. R.; Pierini, M.; Pilati, T.; Rizzo, S.; Sannicolò, F. Eur. J. Org. Chem. 2013, 2013, 8174-8184.

(19) Muñoz, M. P.; Méndez, M.; Nevado, C.; Cárdenas, D. J.; Echavarren, A. M. Synthesis 2003, 2898-2902.

(20) Procedure according to: Kuranaga, T.; Sesoko, Y.; Sakata, K.; Maeda, N.; Hayata, A.; Inoue, M. J. Am. Chem. Soc. 2013, 135, 54675474. 